\title{
Singularity formation in the strongly nonlinear wide-vortex/Tollmien-Schlichting-wave interaction equations
}

\author{
By ANDREW G. WALTON AND RUPA A. PATEL \\ Department of Mathematics, Imperial College of Science, Technology \& Medicine, \\ 180 Queen's Gate, London SW7 2BZ, UK
}

(Received 23 August 1997 and in revised form 9 July 1999)

A combined numerical/analytical study of the wide-vortex/wave interaction equations, describing boundary-layer instability, is presented. Depending on the obliqueness $\beta$ of the wave input, different solution properties are obtained. For $\beta=1$, oscillations in the wave amplitude lead to the evolution of a strongly three-dimensional mean flow, while for $\beta=2$ the interaction is characterized by the development of a singularity in the wave pressure amplitude. This latter behaviour is modelled using an approximate form for the mean flow skin friction and the resulting amplitude equation is analysed using a combination of numerical and asymptotic techniques. A simple method is described for determining the singularity location for a given spanwise wavenumber, and the asymptotic behaviour of the pressure amplitude as the singularity is approached is deduced.

\section{Introduction}

The problem of explaining the circumstances under which laminar-turbulent transition occurs has been occupying scientists since the fundamental experiments of Reynolds (1883). A linearized treatment of the governing Navier-Stokes equations yields results that are in remarkable agreement with experiment (e.g. Healey 1995). Indeed the current state-of-the-art transition prediction tool, the $\mathrm{e}^{N}$ method, is based entirely on a linear approach to the problem. Clearly however, there is a need to develop methods of prediction based on a more rational approach, and this leads inevitably to the consideration of the role of nonlinearity in the transition process. Once the critical importance of nonlinearity is acknowledged there appear at present to be three main approaches in which such effects are incorporated. The first is direct numerical simulation of the Navier-Stokes equations (DNS). Even with the power of modern day computing facilities, results are difficult to obtain, particularly at high Reynolds number, and the physically unrealistic assumption of streamwise periodicity is still sometimes imposed. In addition, since all the terms in the equations are included everywhere in the flow field it can be difficult to decide what aspects of the nonlinearity are important at particular stages in the transition process. Nevertheless some major calculations have been carried out, and much of the work is reviewed in Kleiser \& Zang (1991). More recent work, involving the use of spatial boundary conditions, is described in Reed (1994).

A second approach which has become popular over the last decade is the study of the parabolized stability equation (PSE). In essence the method extends the linear Orr-Sommerfeld equation into the nonlinear regime. Unfortunately this approach, 
when applied to non-parallel base flows, suffers from the same defect as the OrrSommerfeld equation itself, namely that the Reynolds number is assumed finite in some places in the analysis and asymptotically large elsewhere. In addition the method requires the imposition of an irrational shape factor assumption (usually imposed in the form of an integral constraint) which is necessary in order that the evolution of growing modes can be followed. Leaving aside these mathematical objections the method appears to have had some success in predicting transition location for a wide variety of problems, and results compare well with DNS calculations. For more information the reader is referred to the review of Herbert (1997).

The third nonlinear approach to boundary-layer transition is to assume that the Reynolds number is asymptotically large. This is a natural assumption to make given that a formal boundary-layer structure only exists in a mathematical sense at large Reynolds number. Nonlinear effects can then be included in a self-consistent manner with the Reynolds number scalings dependent upon the location at which nonlinear effects are deemed to become important. In the absence of nonlinearity, a small fixed-frequency disturbance propagating through a boundary layer initially decreases in amplitude until the lower-branch neutral location is reached. A region of exponential growth is experienced until the upper neutral location is encountered, and the disturbance ultimately decays. If however the disturbance is amplified sufficiently whilst in the vicinity of the lower neutral point, the rest of the linear neutral curve is irrelevant and the disturbance becomes governed by nonlinear dynamics. In such circumstances the vortex/Tollmien-Schlichting wave interaction (VWI) considered in detail later in this paper is felt to be relevant. In its strongest form it relies on a long scale-short scale interplay between the near-neutral disturbance and the mean flow which evolves in such a way that the wave remains neutral as it increases in amplitude. Although the assumption that it is the neutral waves that play the crucial role in the transition process is often regarded as a limitation of the theory, it is worth noting that every rational nonlinear asymptotic approach is based on some form of small growth rate assumption and is therefore in essence a near-neutral analysis.

If the wave is not amplified sufficiently in the vicinity of the lower-branch neutral point, the upper-branch stability properties become relevant. The instability problem can then either be formulated as a high-frequency limit of a lower-branch analysis or can be based on the upper-branch scaling. In both cases vortex-wave interaction equations can be formulated (e.g. Stewart \& Smith 1992; Wu, Stewart \& Cowley 1996). For more details concerning these interactions and how they are modified in the presence of an adverse pressure gradient the reader is referred to Cowley $\& \mathrm{Wu}$ (1994).

Our concern in the remainder of this paper is in the detailed solution properties of the vortex/Tollmien-Schlichting interaction near the lower branch. The governing equations are similar in many ways to those relating to the PSE approach described earlier, in that both sets of equations exhibit weak ellipticity but are solved by a marching process in which the evolution of the disturbance wavenumber with downstream distance is calculated as part of the solution. The crucial difference between the PSE and VWI approaches, namely that the wave remains neutral in the latter case, means that the lengthscale disparity in the problem remains intact, negating the need for any shape factor assumption. The concept of VWI can be found in papers by Benney (1984) and Benney \& Chow (1985), but the first major VWI study was that of Hall \& Smith (1988). They investigated a channel flow already destabilized by the effects of surface curvature, and showed that the threedimensionality of the mean flow was enhanced by its interaction with a pair of oblique 
Tollmien-Schlichting (TS) modes. In their work, Hall \& Smith found that provided the wave input was sufficiently large, an interaction could take place in the absence of curvature. Motivated by this discovery, Hall \& Smith (1989) (for oblique wave input) and Smith \& Walton (1989) (for near-planar disturbances) considered the analogous interactions in boundary layers. Corrections to some of the details in these papers are presented in Smith \& Blennerhassett (1992), extensions to include compressible effects can be found in Blackaby (1994), while the effect of cross-flow is considered in Davis \& Smith (1994).

Depending on the orders of magnitude of quantities such as the wave amplitude and its spanwise scaling, various strengths of interaction can be formulated. The most powerful is the so-called 'Type III' interaction (Smith \& Walton 1989). In this scenario, the mean flow can be completely altered by the presence of a small-amplitude three-dimensional TS wave in a sublayer of the boundary layer. A crucial part of the authors' analysis is that the wave amplitude and wavenumber are allowed to vary over the lengthscale associated with the development of the mean flow. This is in contrast to classical weakly nonlinear near-neutral analyses (e.g. Smith 1979), in which the carrier wave's properties are assumed to vary only slightly with downstream distance. Extending the work of Smith \& Walton, Hall \& Smith (1991) considered the limiting case of the Type III interaction, where the region over which the mean flow is completely altered occupies the entire boundary layer. The downstream evolution lengthscale then merges with the scale over which the Blasius boundary layer develops in a non-parallel fashion. Hall \& Smith referred to this case as the 'wide vortex'. It is the properties of this particular interaction which will be our main concern in this paper.

To complete our account of the history of VWI, we should mention that in their paper of 1991, Hall \& Smith also formulated the corresponding interaction with Rayleigh waves (vortex-Rayleigh interaction). This interaction, which may be the dominant instability mechanism in the presence of an inflectional mean profile, has been the focus of a number of subsequent papers including Smith, Brown \& Brown (1993), Wu, Lee \& Cowley (1993), Goldstein \& Wundrow (1995) and Brown \& Smith (1996).

The ideas of VWI appear to be generally supported both by experimental evidence (e.g. Aihara, Tomita \& Ito 1984), and numerical simulations (e.g. Wray \& Hussaini 1984). These studies show the development of pronounced three-dimensionality from an initially weak three-dimensional input. Although one can argue about which particular interaction is the most relevant to boundary-layer transition, the ability of the wide vortex to completely alter the Blasius boundary layer via the introduction of remarkably small three-dimensional TS disturbances undoubtedly makes it a powerful contender. The governing interaction equations for the wide vortex are strongly nonlinear and three-dimensional, so that a full numerical solution is necessary in order to shed light on its properties, and to determine how it develops downstream of the position of wave input.

In $\S 2$ of this paper, after introducing the governing equations, we present the results of such a numerical study. The starting conditions imposed are those of a Blasius boundary layer subject to two oblique TS modes with the same frequency and streamwise wavenumber but with equal and opposite spanwise wavenumbers. One of the key features of the results is that for certain spanwise wavenumbers the interaction terminates relatively rapidly (in a spatial sense) in a finite-distance singularity. In $\S 3$ we review the work of Walton, Bowles \& Smith (1994) who solved numerically a simplified version of the wide vortex and obtained similar results. The 
remainder of the paper is devoted to gaining a better understanding of how and why the singularity process occurs. To this end, in $\S 4$ we propose a model for the mean flow part of the interaction. Our model interaction produces results which agree closely with the numerical results of $\S \S 2$ and 3. In $\S \S 5$ and 6 the model interaction is analysed analytically using a perturbation approach in which the distance of formation of the singularity from that of wave input is assumed small. The results of the perturbation method agree well with those obtained numerically and provide a means of visualizing the singularity in a geometrical context. In $\S 7$ we discuss how our methods can be applied to other flow situations and finally in $\S 8$ we consider the singularity structure in more detail and draw some conclusions.

\section{The wide-vortex/TS interaction}

\subsection{Governing equations}

In this section our concern is the numerical solution of the wide-VWI equations formulated by Hall \& Smith (1991) and mentioned briefly in $\S 1$. The interaction arises from a long-scale-short-scale interplay between the mean flow and a smallamplitude three-dimensional TS wave. In order to arrive at the governing equations the Reynolds number $R e$ is taken to be an asymptotically large parameter and the flow velocities, pressure and lengths are scaled with respect to this parameter. The precise scalings required depend upon the flow under consideration, e.g. whether it is incompressible or compressible, internal or external. In this section we will give the scalings relevant to an incompressible boundary layer in an external flow. The scalings for the corresponding interaction in internal flows may be found in Walton (1996), where there is also more discussion concerning the formulation of the equations. We now state the governing equations.

The vortex or mean flow is governed by the steady three-dimensional boundarylayer equations

$$
\left.\begin{array}{c}
\frac{\partial u}{\partial x}+\frac{\partial v}{\partial y}+\frac{\partial w}{\partial z}=0, \\
u \frac{\partial u}{\partial x}+v \frac{\partial u}{\partial y}+w \frac{\partial u}{\partial z}=U_{e}(x) U_{e}^{\prime}(x)+\frac{\partial^{2} u}{\partial y^{2}}, \\
u \frac{\partial w}{\partial x}+v \frac{\partial w}{\partial y}+w \frac{\partial w}{\partial z}=\frac{\partial^{2} w}{\partial y^{2}} .
\end{array}\right\}
$$

In these equations, $u, v$ and $w$ are respectively the streamwise velocity, $R e^{1 / 2}$ times the vertical velocity and $R e^{3 / 8}$ times the spanwise velocity in the boundary layer driven by the external velocity $U_{e}(x)$. The streamwise and normal coordinates are $x$ and $R e^{-1 / 2} y$ as in classical boundary-layer theory, while the spanwise scaling is $R e^{-3 / 8} z$. The latter scaling is chosen so as to be comparable with that for a three-dimensional TS wave near the lower branch of the neutral stability curve. Note that in (2) there is no contribution from the spanwise pressure gradient. These equations are to be solved subject to the boundary conditions

$$
\begin{gathered}
u=v=0, \quad w=w_{s}(x, z) \quad \text { on } \quad y=0, \\
u \rightarrow U_{e}(x), \quad w \rightarrow 0 \quad \text { as } \quad y \rightarrow \infty .
\end{gathered}
$$

In contrast to classical boundary-layer theory there is a spanwise slip velocity $w_{s}(x, z)$. This effect is caused by the self-interaction of three-dimensional TS waves in a 
sublayer of the boundary layer (the lower deck). Hall \& Smith (1991) show that this slip velocity is related in a nonlinear fashion to the wave pressure within the lower deck (see equation (5) below). The wave pressure itself is represented in the form

$$
R e^{-3 / 8}\left(\ln R e^{1 / 8}\right)^{-1 / 2} q(x) p(x, z) \exp \left(\mathrm{i} R e^{3 / 8} \int \alpha(x) \mathrm{d} x-\mathrm{i} R e^{1 / 4} \Omega t\right)+\text { c.c. }
$$

where $\alpha(x)$ and $\Omega$ are the scaled wavenumber and frequency, $t$ is non-dimensional time, c.c. denotes complex conjugate and $q(x)$ is the complex wave amplitude with $p(x, 0)$ taken equal to 1 . The Reynolds number scalings presented here indicate that we are considering the interaction to take place in the vicinity of the lower branch of the neutral curve at high Reynolds number, while the logarithmic factor takes into account the growth of the spanwise velocity within the lower deck. The appropriate relation between the wave pressure and the spanwise slip velocity is

$$
w_{s}(x, z)=-\frac{|q|^{2}}{\alpha^{2} \lambda^{2}} \frac{\partial}{\partial z}\left(\alpha^{2} p p^{*}+\frac{\partial p}{\partial z} \frac{\partial p^{*}}{\partial z}\right) ; \quad \lambda(x, z)=\left.\frac{\partial u}{\partial y}\right|_{y=0},
$$

where $*$ denotes complex conjugate. The equations discussed so far demonstrate the effect of the wave on the mean flow via the spanwise slip condition (5). We now turn to the effect of the mean flow on the wave. The wave pressure is governed by the equation

$$
\frac{\partial^{2} p}{\partial z^{2}}-\frac{1}{\lambda} \frac{\partial \lambda}{\partial z} \mathscr{F}(s) \frac{\partial p}{\partial z}-\alpha^{2} p=(\alpha \lambda)^{5 / 3} \mathscr{G}(s) A(x, z)
$$

where

$$
s(x, z)=\frac{\Omega}{(\alpha \lambda)^{2 / 3}},
$$

together with the law relating the wave pressure $p$ to the wave displacement $-A(x, z)$. In the incompressible regime considered here this law can be derived from the solution of a Helmholtz-type problem in the upper deck of the triple-deck structure. For the purposes of the numerical work to be presented later it is convenient to write this law in the form

$$
\begin{aligned}
& A(x, z)=\frac{\beta}{2 \alpha \pi} \int_{0}^{2 \pi / \beta} p(x, \xi) \mathrm{d} \xi+\frac{\beta}{\alpha^{2} \pi} \sum_{n=1}^{\infty}\left(n^{2} \beta^{2}+\alpha^{2}\right)^{1 / 2} \\
& \times\left(\left\{\int_{0}^{2 \pi / \beta} p(x, \xi) \cos n \beta \xi \mathrm{d} \xi\right\} \cos n \beta z+\left\{\int_{0}^{2 \pi / \beta} p(x, \xi) \sin n \beta \xi \mathrm{d} \xi\right\} \sin n \beta z\right),
\end{aligned}
$$

where $2 \pi / \beta$ represents the spanwise period of the wave. In the pressure equation (6), the coefficients $\mathscr{F}$ and $\mathscr{G}$ can be calculated from the solutions of the following differential equations:

$$
\left.\begin{array}{ll}
\mathscr{F}(s)=\frac{3}{2}+\operatorname{sh}(s) ; & h^{\prime \prime}(s)=\mathrm{i}\left(\frac{1}{2}+s h(s)\right)+\frac{2\left(s h^{\prime}(s)-h(s)\right) h^{\prime}(s)}{\frac{1}{2}+s h(s)}, \\
h(0)=-\mathrm{i}^{1 / 3} \frac{\operatorname{Ai}^{\prime}(0)}{2 \operatorname{Ai}(0)}, & h^{\prime}(0)=\frac{1}{2} \dot{1}^{2 / 3}\left(\frac{1}{3 \operatorname{Ai}(0)}-\left(\frac{\operatorname{Ai}^{\prime}(0)}{\operatorname{Ai}(0)}\right)^{2}\right) ;
\end{array}\right\}
$$




$$
\left.\begin{array}{c}
\mathscr{G}^{\prime \prime}(s)=-\mathrm{i}(\mathscr{G}(s)+s) \mathscr{G}(s)+\frac{2\left(\mathscr{G}^{\prime}(s)\right)^{2}+\mathscr{G}^{\prime}(s)}{\mathscr{G}(s)+s}, \\
\mathscr{G}(0)=-\frac{3 \mathrm{Ai}^{\prime}(0)}{\mathrm{i}^{1 / 3}}, \quad \mathscr{G}^{\prime}(0)=9 \operatorname{Ai}^{\prime}(0) \operatorname{Ai}(0) .
\end{array}\right\}
$$

Here Ai denotes the Airy function. Equation (6) describes how the mean flow affects the wave through the occurrence of the streamwise mean flow skin friction $\lambda(x, z)$ both explicitly and via its presence in the quantity $s$. The governing equations for the wide-VWI are now complete. Our task is to solve from some suitable starting condition, (1)-(5) together with (6)-(10) under the assumption that the wavenumber $\alpha(x)$ remains real. This amounts to supposing that the flow becomes sufficiently nonlinear close to the lower-branch neutral point, with the neutral wave adjusting to the precise amplitude required to provoke and maintain the nonlinear interaction. The complicated nature of these equations clearly indicates that a numerical approach is required, and a suitable algorithm is discussed below.

\subsection{Numerical method}

\subsubsection{The numerical solution of the three-dimensional boundary-layer equations}

We begin by discussing the method of solution of the equations for the mean flow (1), (2) subject to the wall and outer conditions (3), (4). First the velocity components are expanded in a Fourier series in the spanwise direction so that, for example, the streamwise velocity component is written in the form

$$
u=u_{0}(x, y)+\sum_{n=1}^{N} u_{n}(x, y) \mathrm{e}^{\mathrm{i} n \beta z}+\text { c.c. }
$$

The modal truncation number $N$ is determined largely by examining how the results change as $N$ is increased (and also by the available computing resources). For most of the computations presented here we took $N=4$, although some runs were carried out with a truncation number of 8 .

The velocity expansions are substituted into the momentum equations (2), yielding $2 N+1$ nonlinear differential equations (first order in $x$, second order in $y$ ) for each of the unknowns $u_{n}, w_{n}$ and their complex conjugates. There are an additional $2 N+1$ equations arising out of the continuity equation (1). As an example, the equation for $u_{0}$ takes the form

$$
\frac{\partial^{2} u_{0}}{\partial y^{2}}=-U_{e} U_{e}^{\prime}+v_{0} \frac{\partial u_{0}}{\partial y}+u_{0} \frac{\partial u_{0}}{\partial x}+\sum_{k=1}^{N}\left(v_{k}^{*} \frac{\partial u_{k}}{\partial y}+\mathrm{i} k \beta w_{k}^{*} u_{k}+u_{k}^{*} \frac{\partial u_{k}}{\partial x}+\text { c.c. }\right) .
$$

These equations are solved by finite differences in $x$ and $y$, with associated step lengths $\Delta x, \Delta y$. We used a fully implicit scheme in the streamwise direction so that the discretized version of (11) can be written as

$$
a_{0, j} u_{0, j-1}^{m+1}+b_{0, j} u_{0, j}^{m+1}+c_{0, j} u_{0, j+1}^{m+1}+\sum_{k=1}^{N}\left(a_{k, j} u_{k, j-1}^{m+1}+b_{k, j} u_{k, j}^{m+1}+c_{k, j} u_{k, j+1}^{m+1}+\text { c.c. }\right)=d_{j}
$$

where the coefficients are given by

$$
a_{0, j}=-\frac{\Delta x}{(\Delta y)^{2}}-\frac{1}{2} \frac{\Delta x}{\Delta y} v_{0, j}^{m+1}, b_{0, j}=2 \frac{\Delta x}{(\Delta y)^{2}}+u_{0, j}^{m+1}, c_{0, j}=-\frac{\Delta x}{(\Delta y)^{2}}+\frac{1}{2} \frac{\Delta x}{\Delta y} v_{0, j}^{m+1},
$$




$$
\begin{aligned}
a_{k, j} & =-\frac{1}{2} \frac{\Delta x}{\Delta y} v_{k, j}^{m+1 *}, b_{k, j}=\mathrm{i} k \beta(\Delta x) w_{k, j}^{m+1^{*}}+u_{k, j}^{m+1^{*}}, c_{k, j}=\frac{1}{2} \frac{\Delta x}{\Delta y} v_{k, j}^{m+1^{*}}, \\
d_{j} & =\left(U_{e} U_{e}^{\prime}\right)^{m+1}(\Delta x)+u_{0, j}^{m+1} u_{0, j}^{m}+\sum_{k=1}^{N}\left(u_{k, j}^{m+1^{*}} u_{k, j}^{m}+\text { c.c. }\right) .
\end{aligned}
$$

In the above expressions a superscript denotes $x$-location, while the second subscript denotes $y$-location. At each $x$-step a block tridiagonal system of equations needs to be solved; in view of the implicit nature of the scheme, a sequence of iterations is performed at each downstream location until convergence of the solution to some tolerance is achieved. The set of equations for $u_{n}$ were solved first, followed by those for $w_{n}$ and then the $v_{n}$ were determined from the discretized version of the continuity equation. This process was then repeated. In practice we found that ten iterations were sufficient to guarantee convergence to seven decimal places.

The main reason for using an implicit scheme is that the effect of the wave via the spanwise slip condition (5) can then be transmitted to the mean flow at the first $x$-step because of the occurrence of terms such as $w_{k, j}^{m+1}$ in (12) and the analogous equations for the $u_{n}$ and $w_{n}$. This enables the generation of a three-dimensional interaction from an initially two-dimensional mean flow: see below for a discussion of starting conditions. The disadvantage of this differencing method is that the scheme is only first-order accurate in $x$, so that a small streamwise step length $\Delta x$ is required in order to obtain accurate solutions. This is not a major drawback however, since the use of a small $x$-step proved necessary anyway in order to follow the evolution of the solution which in some instances developed rapidly in the streamwise direction. An alternative to using the implicit scheme would be to use the starting solution derived in Walton (1996) at the first $x$-step.

\subsubsection{Numerical solution of the pressure equation}

We now turn to the treatment of the pressure equation (6). This is solved using centred finite differences in the spanwise direction. In most of the computations we performed we took 5000 points between $z=0$ and $z=\pi / \beta$. The functions $\mathscr{F}$ and $\mathscr{G}$ are determined numerically from the differential equations (9), (10), while the righthand side of (6) was split between old and new $x$-locations with $A$ evaluated using the series form (8). The integrals present in (8) were computed using Simpson's rule. The appropriate boundary conditions to apply to this equation are a normalization of the form

and a symmetry condition

$$
p(x, 0)=1
$$

$$
\frac{\partial p}{\partial z}\left(x, \frac{\pi}{\beta}\right)=0
$$

For more details concerning the numerical approach to solving (6), the reader is referred to Walton et al. (1994) and Patel (1997).

\subsubsection{Solution procedure for the full system}

The two schemes described above are combined and the solution is advanced from one $x$-step to the next in the following manner. First a guess is made for the pressure amplitude $|q|$ (usually the value obtained at the previous $x$-step will suffice). Next the spanwise slip velocity $w_{s}$ is evaluated from (5) using the old values of pressure and wavenumber. Then the boundary-layer equations (1), (2) are solved by the method described above, yielding a prediction for the new skin-friction distribution $\lambda(x, z)$. 

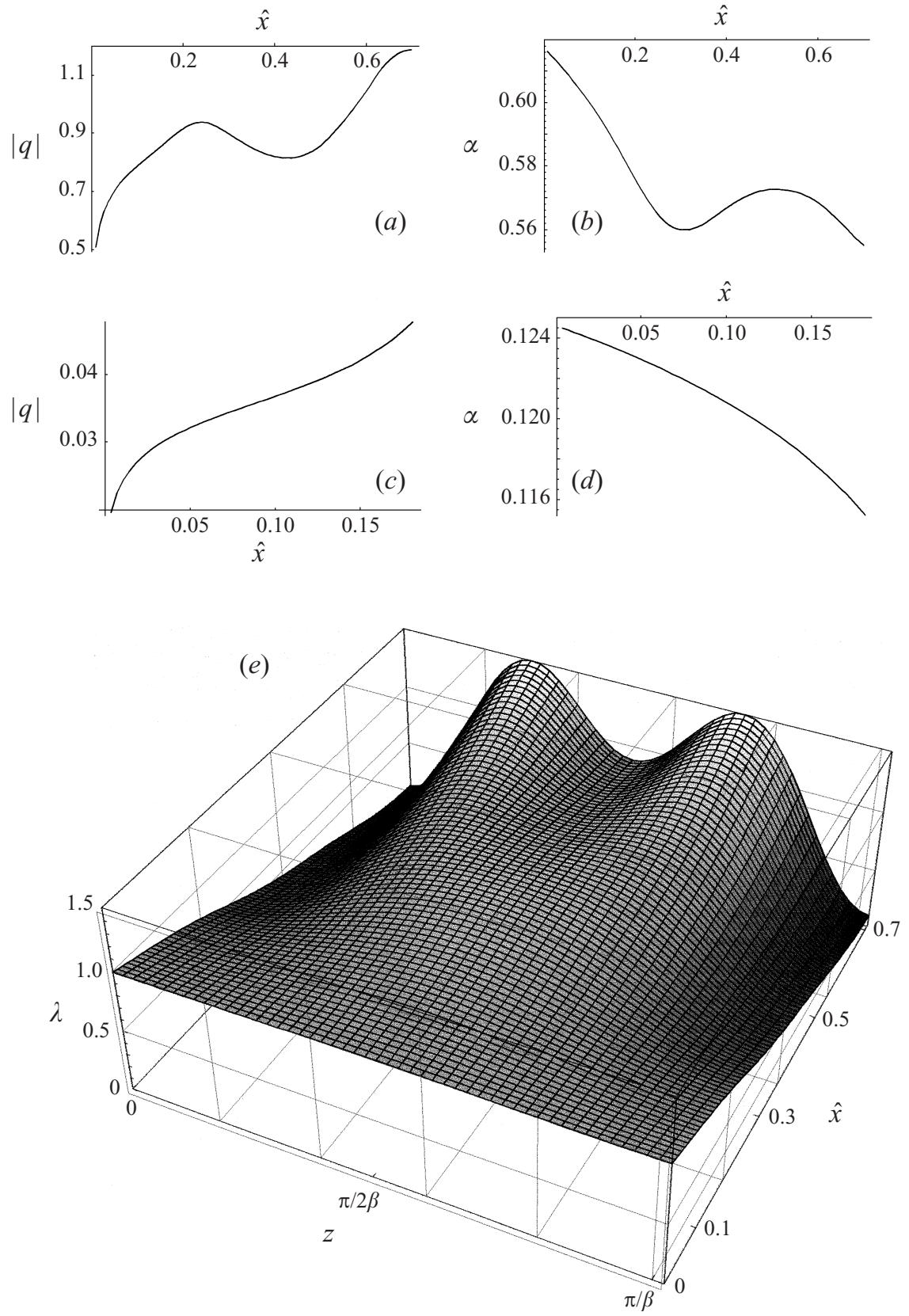

FIGURE $1(a-e)$. For caption see facing page.

This is substituted into the pressure equation (6), both explicitly and via the function $s$, and (6) is solved as described above subject to the conditions

$$
p(x, 0)=1, \quad \frac{\partial p}{\partial z}(x, 0)=0 .
$$

In general the symmetry condition (13) will not be satisfied: in order to overcome this we apply Newton iteration to the real quantities $\alpha$ and $\Omega$ until symmetry is attained. 


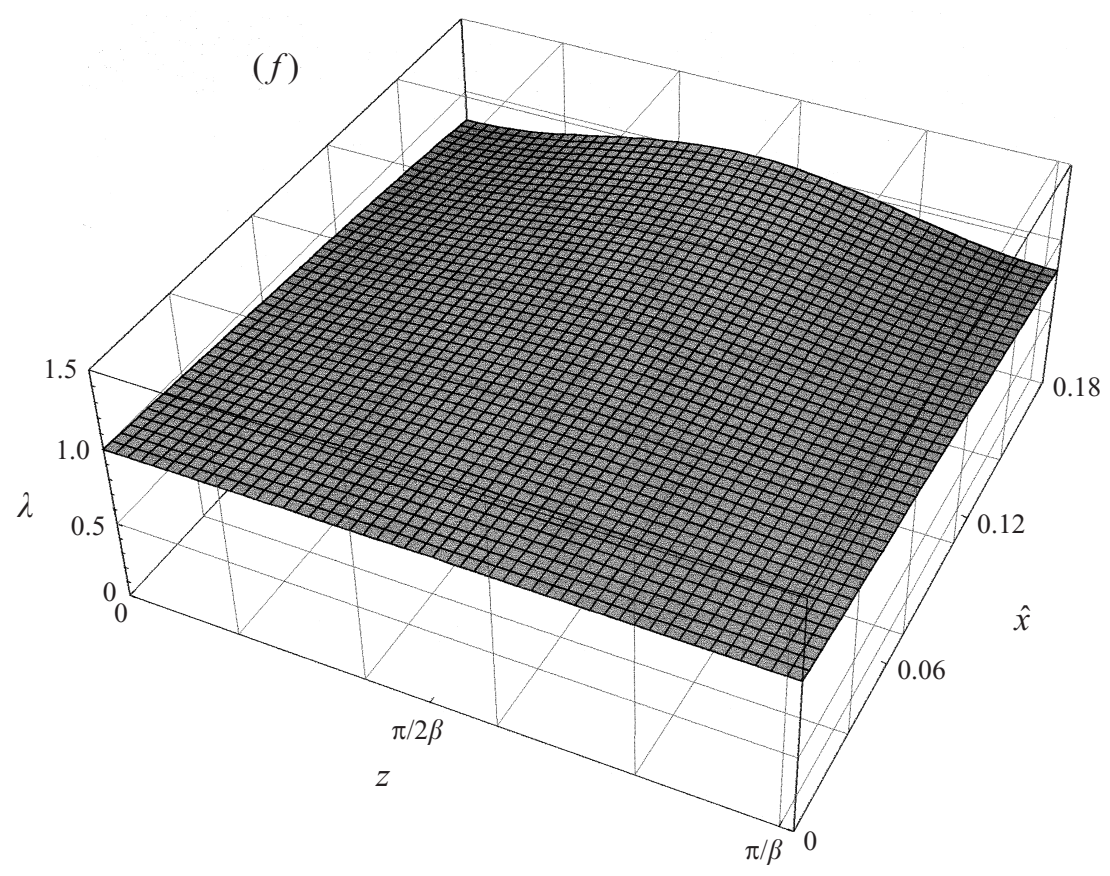

FIGURE 1. Numerical results for the wide VWI of $\S 2$. (a) Pressure amplitude $|q|$ versus distance from wave input $\hat{x}$ for spanwise wavenumber $\beta=1$. (b) Streamwise wavenumber $\alpha(\hat{x})$ for $\beta=1$. (c) $|q(\hat{x})|$ for $\beta=2$. (d) $\alpha(\hat{x})$ for $\beta=2$. (e) Evolution of mean flow skin friction $\lambda(\hat{x}, z)$ for $\beta=1$. (f) $\lambda(\hat{x}, z)$ for $\beta=2$.

Since the mean flow is time-independent however, the frequency of the wave must remain equal to its neutral starting value for all $x$. This is achieved by an outer iteration upon the wave amplitude $|q|$, each iteration requiring us to repeat the entire process described above. Once this iteration has converged we have obtained the wavenumber, pressure and the velocity components at the new $x$-station.

\subsection{Starting conditions}

The only previous attempt at a numerical solution of the full equations was that carried out by Hall \& Smith (1991). Their starting conditions consisted of a somewhat arbitrary weakly three-dimensional mean flow together with an almost two-dimensional TS wave. The scheme adopted here allows us to start from a purely two-dimensional mean flow, a situation that could be simulated in the laboratory and is certainly less arbitrary. In all the computations reported here we imposed a uniform external outer flow and used the Blasius profile as our starting mean flow at the location $x=x_{0}$ say. A spanwise wavenumber $\beta$ is selected and the corresponding starting wavenumber $\alpha\left(x_{0}\right)$ and neutral frequency are determined from the linear TS eigenrelation to which (6) reduces when the skin friction $\lambda$ is independent of $z$. The wave pressure $p$ is taken to have a starting form proportional to $\cos \beta z$ and the starting amplitude $q\left(x_{0}\right)$ may be taken to be zero. The solution is then advanced to the next $x$-location $x_{1}$ say, in the manner described in the previous subsection, and in particular the value of $\left|q\left(x_{1}\right)\right|$ is determined. The initial development of the interaction from these starting conditions can be described analytically and the reader is referred to Walton (1996) for more details. 
Such a starting condition might perhaps be realized in a carefully-controlled experiment by using a corrugated strip to preferentially excite TS waves of a certain spanwise wavenumber. This type of disturbance generation has been used in the experiments of Kovasznay, Komoda \& Vasudeva (1962) and Klebanoff, Tidstrom \& Sargent (1962), although the waves generated in the former case had longer scales than the triple-deck ones envisaged here.

\subsection{Numerical results}

In figure 1 we present the variation of the pressure amplitude $|q|$ and wavenumber $\alpha$ with distance from wave input $\hat{x}\left(\equiv\left(x-x_{0}\right) / x_{0}\right)$ for the spanwise wavenumbers $\beta=1$ and $\beta=2$. The results shown here are for a vertical step size $\Delta y=0.02$. In both cases we started from an input location at which the Blasius profile has a wall shear of unity. First we comment on the case $\beta=1$, where the streamwise step length $\Delta x=0.001$. Figure $1(a)$ shows that $|q|$ initially grows in a linear fashion but then the rate of growth is inhibited by nonlinear effects. A maximum in $|q|$ is reached at $\hat{x} \approx 0.2$ followed by a decrease until $\hat{x} \approx 0.4$, at which point it starts to increase again. This oscillatory behaviour, with an overall increasing trend, continues until the computational time expires. A similar oscillatory behaviour (this time with a decreasing trend) is observed in the graph of $\alpha(\hat{x})$ (figure $1 b$ ). We also present the evolution of the skin friction from its initial uniform state (figure 1e). Here we see that the mean flow is becoming strongly three-dimensional. It seems likely that if the computation were allowed to run for a sufficiently long time, $\lambda$ would eventually touch zero at some spanwise location. At this point the governing equations would no longer be valid. It is more likely however that the flow would lose stability to some other form of disturbance (viscous or inviscid in nature) well before this point is reached-the computations show that the mean flow becomes inflectional quite rapidly, with the inflection point moving away from the wall as $x$ increases. Nevertheless, we believe that this computation is significant since it demonstrates that an interaction between a two-dimensional flow and a three-dimensional TS wave is self-sustaining and can result in a strongly three-dimensional mean flow downstream.

Turning now to the case $\beta=2$, we observe quite different behaviour. We were unable to find a solution for $|q|$ beyond $\hat{x} \approx 0.18$ despite making refinements to the grid close to this location (our most refined calculations have $\Delta x=10^{-4}$ ). There is no sign of any oscillations in the solution for $|q|$ (figure $1 c$ ) or for the wavenumber $\alpha$ (figure $1 d$ ), in contrast to the behaviour observed for $\beta=1$. It is our opinion that for the case $\beta=2,|q|$ becomes infinite at the critical $x$-location, although the numerical evidence is not entirely conclusive. We will return to this point in later sections. A similar singularity was found by Hall \& Smith (1991) in their numerical solutions although, as remarked earlier, their starting conditions were somewhat different to ours. Figure $1(f)$ shows that three-dimensionality in the skin friction appears to develop at a similar rate to the case of $\beta=1$. It therefore possesses only weak spanwise variations at the location where the pressure amplitude apparently becomes singular in this case.

A significant part of the remainder of this paper is devoted to gaining an understanding of why a singularity should arise for certain spanwise wavenumbers and not others. This form of blow-up of the pressure amplitude was also observed by Walton et al. (1994) in their study of vortex-wave interaction under the influence of a strong adverse pressure gradient. We review their work in the next section. 


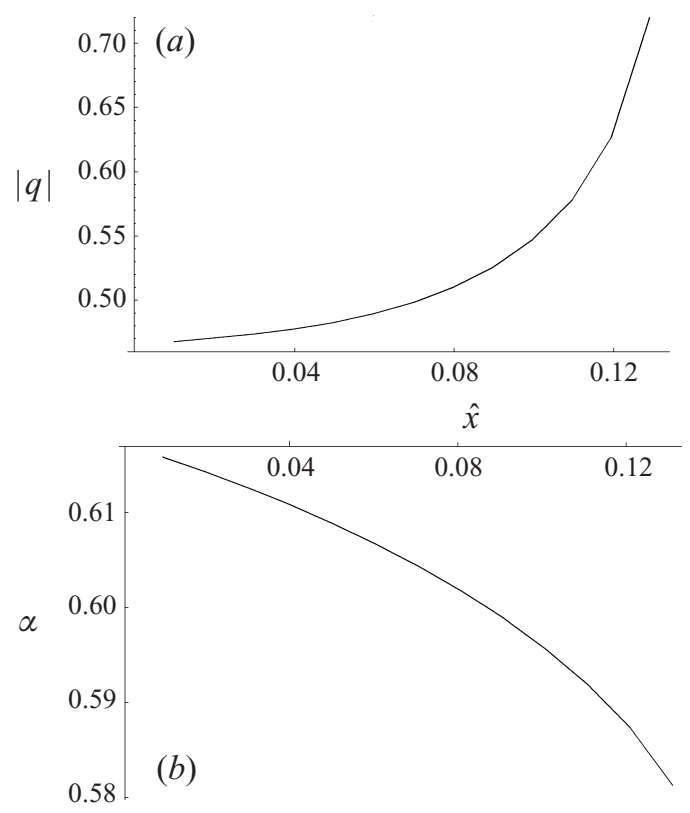

FIGURE 2. Numerical results for a VWI subject to a strong adverse pressure gradient ( 3 ).

(a) Pressure amplitude $|q(\hat{x})|$ for $\beta=1$. (b) Wavenumber $\alpha(\hat{x})$ for $\beta=1$.

\section{The wide-VWI subject to a strong adverse pressure gradient}

3.1. Governing equations and numerical results

It is of some interest to compare the numerical results of $\S 2$ with those obtained by Walton et al. (1994, henceforth referred to as WBS) for the case of a VWI subject to a strong adverse pressure gradient. We will first write down the governing equations and then discuss their numerical solution. WBS consider the case where the boundary-layer flow is driven by an external pressure gradient of the form

$$
U_{e}(x) U_{e}^{\prime}(x)=-K+O(\Delta)
$$

with $\Delta \ll 1$ and $K$ a positive constant. The wide-VWI equations of $\S 2$ can then be analysed on an $O(\Delta)$ length scale about the location of zero skin friction. WBS find that the pressure equation (6) and the spanwise slip condition (5) remain unchanged, but that (1), (2) for the mean flow are replaced by the single equation for the skin friction,

$$
\lambda(x, z) \frac{\partial \lambda}{\partial x}=-\frac{1}{2}+\frac{\partial}{\partial z} w_{s}(x, z) .
$$

In the absence of any wave forcing the classical square-root singularity in the skin friction is encountered at $x=0$ (Goldstein 1948). The numerical task then, is to solve (14) together with (5), (6) starting from an initial condition of undisturbed skin friction, namely

$$
\lambda(x, z)=1 \text { at } x=-1
$$

in scaled variables. WBS use a finite-difference approach to solving the system and we used their method to obtain a numerical solution for a spanwise wavenumber $\beta=1$. The results are shown in figure 2. We also carried out a number of computations for other spanwise wavenumbers and obtained similar results to the $\beta=1$ case. The 
main feature of the results is the occurrence of a singularity in the pressure amplitude as some finite $x$-station $x_{s}(\beta)$ is approached. For the cases of $\beta=1$, the singularity occurred at a distance from wave input $\hat{x} \approx 0.13$. For other values of $\beta$, the singularity position was altered slightly but always occurred relatively close to the position of wave input. The results are quite similar to those for $\beta=2$ presented in $\S 2$ : $|q|$ increases monotonically and $\alpha$ decreases monotonically. For this interaction however, there appears to be no case that corresponds to the oscillatory behaviour exhibited by the wide-VWI for $\beta=1$.

In conclusion, both this system and the wide vortex of $\S 2$ appear to share the same type of solution properties. Since both systems contain the wave pressure equation (6), it would appear that in order to gain an understanding of how and why the singularity arises we need to analyse the properties of this equation.

\section{Model profiles for the skin friction}

\subsection{The wide vortex}

Our aim here is to construct a relatively simple form for the skin friction in terms of the pressure amplitude which closely models the behaviour observed in the full numerical simulations of $\S 2$. To begin with we consider the linear response of the system (1)-(6) close to the position of wave input $x=x_{0}$ say. The flow near to the wall will be a small perturbation to the near-wall form of the Blasius flow:

$$
u_{B} \sim \frac{\hat{\lambda}}{x_{0}^{1 / 2}} y+O\left(\frac{x-x_{0}}{x_{0}}\right),
$$

where $\hat{\lambda} \approx 0.332$. Linearizing about the basic shear, equations (1), (2) for the wide vortex reduce to

$$
\frac{\partial^{2} \tau}{\partial y^{2}}-y \frac{\partial \tau}{\partial x}=-\frac{\partial \bar{w}}{\partial z}, \quad \frac{\partial^{2} \bar{w}}{\partial y^{2}}-y \frac{\partial \bar{w}}{\partial x}=0,
$$

where $\tau(x, y, z)=\partial \bar{u} / \partial y$ and $\bar{u}, \bar{w}$ denote the three-dimensional streamwise and spanwise perturbations to the uniform shear. Use of Fourier transforms leads to the result

$$
\tau(x, 0, z)=-\frac{\operatorname{Ai}^{\prime}(0)}{\operatorname{Ai}(0) \Gamma\left(\frac{2}{3}\right)} \int_{x_{0}}^{x} \frac{\partial \bar{w}}{\partial z}(\xi, 0, z) \frac{\mathrm{d} \xi}{(x-\xi)^{1 / 3}} .
$$

Expanding the integral about $x=x_{0}$ and assuming an input pressure proportional to $\cos \beta z$, we find that in the linear regime the skin friction develops according to

$$
\begin{aligned}
\lambda= & \frac{\hat{\lambda}}{x_{0}^{1 / 2}}\left(1-\frac{1}{2}\left(\frac{x-x_{0}}{x_{0}}\right)+\frac{3}{8}\left(\frac{x-x_{0}}{x_{0}}\right)^{2}-\frac{5}{16}\left(\frac{x-x_{0}}{x_{0}}\right)^{3}\right. \\
& \left.+\frac{35}{128}\left(\frac{x-x_{0}}{x_{0}}\right)^{4}+\ldots\right)-\hat{q}\left(\frac{x-x_{0}}{x_{0}}\right) \cos 2 \beta z \\
& + \text { terms of order }\left(\frac{x-x_{0}}{x_{0}}\right)^{2} \text { with no mean part, }
\end{aligned}
$$

where the near-wall form of the Blasius flow has been expanded up to $O\left(x-x_{0}\right)^{4}$ and $\bar{w}$ has been evaluated from (5). The real quantity $\hat{q}$ can be calculated for a given spanwise wavenumber $\beta$ : for more details concerning this point see Walton (1996). It is sufficient for our purposes here to note the form of the skin-friction profile (16). In 
view of this we propose a model profile of the form

$$
\begin{aligned}
\lambda_{M}=\frac{\hat{\lambda}}{x_{0}^{1 / 2}}\left(1-\frac{1}{2}\left(\frac{x-x_{0}}{x_{0}}\right)+\frac{3}{8}\right. & \left(\frac{x-x_{0}}{x_{0}}\right)^{2}-\frac{5}{16}\left(\frac{x-x_{0}}{x_{0}}\right)^{3} \\
& \left.+\frac{35}{128}\left(\frac{x-x_{0}}{x_{0}}\right)^{4}\right)+\frac{1}{2} B(x) \cos 2 \beta z .
\end{aligned}
$$

Some remarks about our choice of profile are appropriate at this stage. Clearly, comparison of (16) and (17) indicates that the initial behaviour of the amplitude function $B$ is of the form $B(x) \propto\left(x-x_{0}\right)$. Later in $\S 6$ we will be seeking a perturbation solution in which $B$ is assumed to remain relatively small. For this purpose we will assume that $B$ remains of order $\left(x-x_{0}\right)$ throughout the interaction. The functions $\mathscr{F}$ and $\mathscr{G}$ in the pressure equation depend nonlinearly on $B$, in view of their nonlinear dependence on $\lambda$ via the quantity $s$ defined in (7). In order to include the effects of nonlinear amplitude dependence in the pressure equation it will prove necessary to retain terms up to order $B^{4}$ (see $\S 6$ ). Thus for the sake of consistency we should also retain terms up to $O\left(x-x_{0}\right)^{4}$. This necessitates expanding the near-wall form for the Blasius flow up to this order as indicated in (17) above. The approximation of $\lambda$ by (17) serves to suppress the creation of higher harmonics by wave action but allows the wave to develop in a nonlinear three-dimensional manner under the influence of the mean flow. This approximation appears to be reasonable since from inspection of the numerical results of $\S 2$, it appears that the generation of harmonics higher than $\cos 2 \beta z$ in the skin friction is extremely weak (see figure $1 f$ ) and is unlikely to be responsible for the development of the singularity observed in the computation for $\beta=2$. In the Appendix we demonstrate that there is a range of values of spanwise wavenumber $\beta$ for which (17) constitutes a rational approximation to the full problem. It is worth noting here that upon the basis of linear theory, the function $B$ plays the role of $\int_{x_{0}}^{x}|q(\xi)|^{2} /(x-\xi)^{1 / 3} \mathrm{~d} \xi$ in the full computations, in view of the result (15).

We now wish to check that our approximation has not changed the dynamics of the problem under consideration. This can be achieved by using the skin-friction profile $\lambda_{M}$ to calculate the coefficients $\mathscr{F}$ and $\mathscr{G}$ via (9), (10), (7) and then solving the pressure equation (6) numerically at different values of $x$. This constitutes an eigenvalue problem in which the real parameters $\alpha$ and $B$ can be determined at a given distance from wave input $\hat{x}$ and spanwise wavenumber $\beta$ (and corresponding neutral frequency $\Omega$ ). The results of these computations are shown in figure 3 for the spanwise wavenumbers $\beta=1,2$. For $\beta=1$ we see that the results for the wavenumber are in good agreement with those for the full computation of $\S 2$ initially, but eventually the behaviours are quite different. Clearly our model skin friction is not a good approximation for this case. This is no surprise since we can see from figure $1(e)$ that $\lambda$ clearly eventually develops harmonics higher than $\cos 2 \beta z$. For $\beta=2$ however, figure $3(c)$ shows that no solution for $B$ exists beyond a critical distance $\hat{x} \approx 0.19$ from the position of wave input. This point is very close to that at which the full computations develop a singularity (see figure $1 c$ ). In addition we see from figure $3(d)$ that the wavenumber evolution is very similar to that for the full computation (figure $1 d$ ) throughout the entire duration of the interaction. In view of the relation between $\mathrm{d} B / \mathrm{d} x$ and $|q|^{2}$ noted above, it is possible for $B$ to remain finite at the location where $|q|$ develops a singularity. It therefore appears that the model profile gives a good approximation to the skin friction that drives the full numerical solution of $\S 2$ for $\beta=2$. In $\S 6$ we use perturbation techniques to find an analytic 

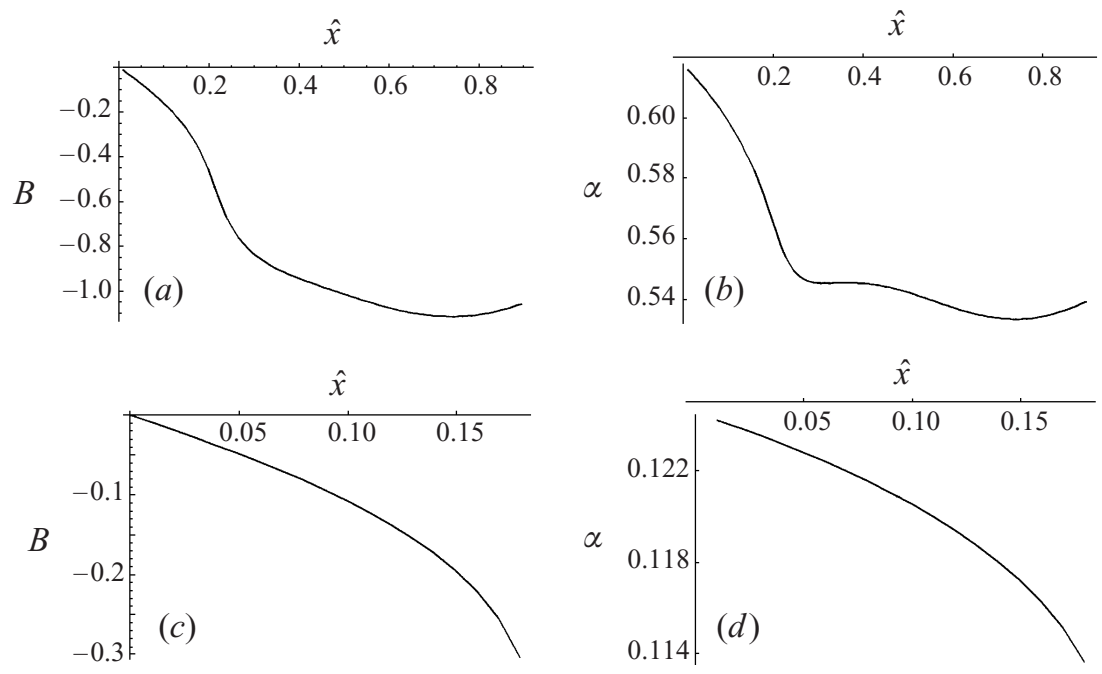

FIGURE 3. The numerical solution of the pressure equation with a model skin friction appropriate to the wide VWI of $\S 2$. (a) Effective amplitude $B(\hat{x})$ for $\beta=1$. (b) $\alpha(\hat{x})$ for $\beta=1$. (c) $B(\hat{x})$ for $\beta=2$. (d) $\alpha(\hat{x})$ for $\beta=2$.

form for $B$ as a function of $x$ and we demonstrate why a solution for $B$ ceases to exist beyond a critical value of $x, x_{c}$ say. In addition we will be able to provide a simple formula for predicting $x_{c}(\beta)$ without recourse to numerical simulations.

\subsection{VWI in a strong adverse pressure gradient}

In a similar fashion to the previous subsection, here we motivate and propose a suitable model skin-friction profile for this slightly simplified form of the wide-VWI. Recall from $\S 3$ that the governing equation for the skin friction is

$$
\lambda \frac{\partial \lambda}{\partial x}=-\frac{1}{2}+\frac{\partial}{\partial z} w_{s}(x, z)
$$

with the spanwise slip velocity given in terms of the pressure amplitude by

$$
w_{s}(x, z)=-\frac{|q|^{2}}{\alpha^{2} \lambda^{2}} \frac{\partial}{\partial z}\left(\alpha^{2} p p^{*}+\frac{\partial p}{\partial z} \frac{\partial p^{*}}{\partial z}\right) .
$$

The pressure evolution equation remains the same, namely (6). Integrating (18) with respect to $x$ we obtain

$$
\lambda^{2}=-x+\int_{-1}^{x} 2 \frac{\partial}{\partial z} w_{s}(\xi, z) \mathrm{d} \xi,
$$

indicating that in the absence of any wave forcing the skin friction will eventually develop a square-root singularity (Goldstein 1948). We are interested in modelling the flow in the presence of a wave close to the position of wave input, normalized to -1 in the above equation. Linear theory (WBS) indicates that for an input wave proportional to $\cos \beta z$, the skin friction develops according to

$$
\lambda^{2}=1+(x+1)(-1+\bar{q} \cos 2 \beta z)+O(x+1)^{2}
$$

where, as in $\S 4.1$, the coefficient $\bar{q}$ can be calculated for a given spanwise wavenumber $\beta$. In view of this linear development we propose the following model profile for the 


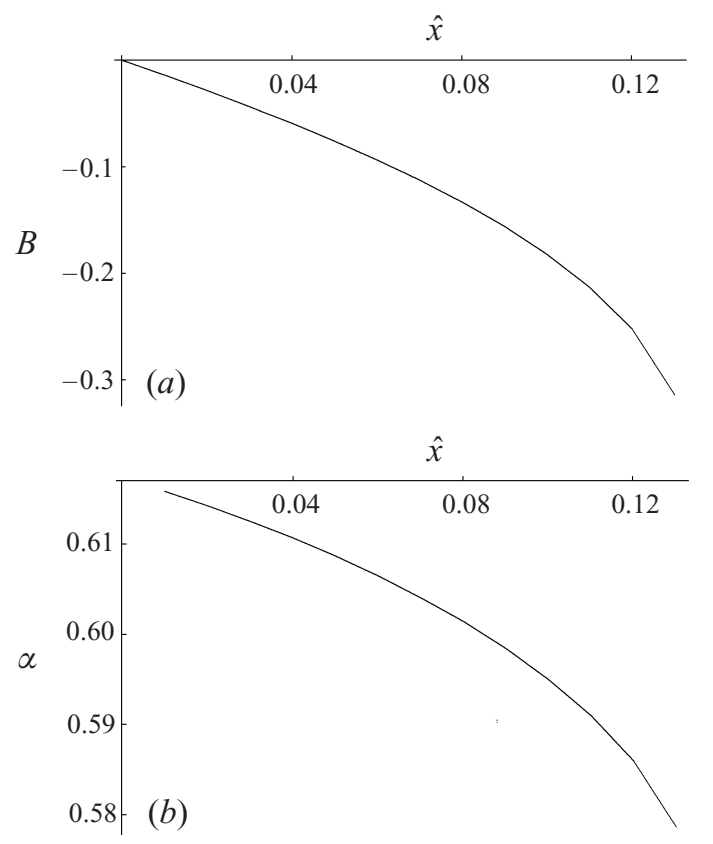

FIGURE 4. The numerical solution of the pressure equation with a model skin friction appropriate to the adverse pressure gradient VWI of $\S 3$. (a) $B(\hat{x})$ for $\beta=1$. (b) $\alpha(\hat{x})$ for $\beta=1$.

skin-friction squared:

$$
\lambda_{M}^{2}=-x+B(x) \cos 2 \beta z
$$

where, by comparison with (19), the function $B$ is seen to be a measure of $\int_{-1}^{x}|q(\xi)|^{2} \mathrm{~d} \xi$. We note that the mean part of this profile is exact to all orders and that once again we have suppressed the creation of higher harmonics by wave interaction. Analysis of the numerical results of $\S 3$ and those of WBS indicates that this process is negligible even close to the singularity location. Once again, the approximation of the skin friction by (20) can be shown to be a rational approximation over a range of spanwise wavenumbers (see Appendix for details). As in $\$ 4.1$ we can demonstrate the practical validity of (20) by solving the pressure equation numerically and calculating $B$ and $\alpha$ as functions of $x$ for various spanwise wavenumbers $\beta$. The results are shown in figure 4 for the case $\beta=1$, the results being similar for other values of $\beta$. Once again the solution for $B$ disappears close to where the singularity forms in the computations of $\S 3$, and the results for $\alpha(\hat{x})$ are in excellent agreement with those presented earlier.

Now that we have chosen our model skin-friction profiles we proceed in the next section to derive a condition for a periodic solution of the pressure equation. This will ultimately enable us to formulate an amplitude equation to be satisfied by $B$ for the two cases considered above.

\section{A perturbation approach to solving the pressure equation}

In $\S \S 2$ and 3 we have seen that numerical simulations of the wide-VWI equations often end with the development of a singularity in the pressure amplitude. In $\S 4$ we saw that replacing the skin friction by a form containing one parameter $B$ gave very similar results. We now wish to understand why this simplified system possesses no solution beyond a critical $x$-station. In order to achieve this we will adopt a 
perturbation approach to the problem and assume that the parameter $B$ remains small for the duration of the interaction. This assumption is not unreasonable since the numerical results of the previous section indicate that the critical value of $|B|$ is in the range 0.3-0.4. In this section we concentrate on developing a condition for a periodic solution of the pressure equation for a given model skin friction $\lambda_{M}$ (either (17) or (20)). To this end we will expand the coefficients of the equation in Fourier series form. Ultimately our approach will make use of the observation from the numerical results that the stoppage occurs relatively close to the position of wave input. To begin, we write out the pressure equation in the form

$$
\begin{aligned}
p^{\prime \prime}-\frac{\lambda^{\prime}}{\lambda} \mathscr{F}(s) p^{\prime}-\alpha^{2} p= & \frac{(\alpha \lambda)^{5 / 3} \mathscr{G}(s)}{\alpha^{2}}\left[\frac{\alpha \beta}{2 \pi} \int_{0}^{2 \pi / \beta} p(x, \xi) \mathrm{d} \xi\right. \\
& +\frac{\beta}{\pi} \sum_{n=1}^{\infty}\left(n^{2} \beta^{2}+\alpha^{2}\right)^{1 / 2}\left(\left\{\int_{0}^{2 \pi / \beta} p(x, \xi) \cos n \beta \xi \mathrm{d} \xi\right\} \cos n \beta z\right. \\
& \left.\left.+\left\{\int_{0}^{2 \pi / \beta} p(x, \xi) \sin n \beta \xi \mathrm{d} \xi\right\} \sin n \beta z\right)\right]
\end{aligned}
$$

where ' denotes differentiation with respect to $z$. We define

$$
G=\frac{(\alpha \lambda)^{5 / 3} \mathscr{G}}{\alpha^{2}}, \quad H=-\frac{\lambda^{\prime}}{\lambda} \mathscr{F}
$$

and expand $G$ and $H$ as

$$
\begin{gathered}
G=G_{0}+G_{2} \cos 2 \beta z+G_{4} \cos 4 \beta z+\ldots, \\
H=H_{2} \sin 2 \beta z+H_{4} \sin 4 \beta z+\ldots .
\end{gathered}
$$

The forms for $G$ and $H$ are motivated by the model skin frictions of $\S 4$. In view of this development for $G$ and $H$, the appropriate expansion for the pressure is

$$
p=\cos \beta z+p_{3} \cos 3 \beta z+p_{5} \cos 5 \beta z+\ldots .
$$

In our perturbation approach it transpires that the coefficients of higher harmonics are negligible at the order to which we take our approximation. The terms denoted by ... in (23), (24), (25) will therefore not be considered further. Our aim now is to obtain a condition for a periodic solution of (21) in terms of the unknown coefficients $G_{0}, G_{2}, G_{4}, H_{2}, H_{4}$. Substituting (23), (24), (25) into the pressure equation (21), and equating coefficients of $\cos \beta z$ we find that

$$
\begin{aligned}
-\beta^{2}- & \alpha^{2}-G_{0}\left(\beta^{2}+\alpha^{2}\right)^{1 / 2}-\frac{1}{2} \beta H_{2}-\frac{1}{2} G_{2}\left(\beta^{2}+\alpha^{2}\right)^{1 / 2} \\
& =\left(\frac{3}{2} \beta H_{2}+\frac{3}{2} \beta H_{4}+\frac{1}{2} G_{4}\left(9 \beta^{2}+\alpha^{2}\right)^{1 / 2}\right) p_{3}+\left(\frac{5}{2} \beta H_{4}+\frac{1}{2} G_{4}\left(25 \beta^{2}+\alpha^{2}\right)^{1 / 2}\right) p_{5} .
\end{aligned}
$$

In order to determine $p_{3}, p_{5}$ we need to equate coefficients of $\cos 3 \beta z, \cos 5 \beta z$ in (21). This gives

$$
\begin{aligned}
\left(G_{0}\left(9 \beta^{2}+\alpha^{2}\right)^{1 / 2}+\right. & \left.9 \beta^{2}+\alpha^{2}\right) p_{3}+\left(\frac{1}{2} G_{2}\left(25 \beta^{2}+\alpha^{2}\right)^{1 / 2}+\frac{5}{2} \beta H_{2}\right) p_{5} \\
& =\frac{1}{2} \beta H_{2}-\frac{1}{2} \beta H_{4}-\frac{1}{2} G_{2}\left(\beta^{2}+\alpha^{2}\right)^{1 / 2}-\frac{1}{2} G_{4}\left(\beta^{2}+\alpha^{2}\right)^{1 / 2},
\end{aligned}
$$

and

$$
\begin{aligned}
\left(G_{0}\left(25 \beta^{2}+\alpha^{2}\right)^{1 / 2}+25 \beta^{2}+\alpha^{2}\right) p_{5}+\left(\frac{1}{2} G_{2}\left(9 \beta^{2}+\alpha^{2}\right)^{1 / 2}-\frac{3}{2} \beta H_{2}\right) p_{3} & \\
& =\frac{1}{2} \beta H_{4}-\frac{1}{2} G_{4}\left(\beta^{2}+\alpha^{2}\right)^{1 / 2} .
\end{aligned}
$$




$\begin{array}{lrll}\mathscr{F}_{0} & 0.1881+1.7484 \mathrm{i} & \mathscr{G}_{0} & -1.0007 \\ \mathscr{F}_{1} & -2.4804-0.9728 \mathrm{i} & \mathscr{G}_{1} & -0.7066+0.5302 \mathrm{i} \\ \mathscr{F}_{2} & 0.8804-2.4022 \mathrm{i} & \mathscr{G}_{2} & -0.1042+0.2753 \mathrm{i} \\ \mathscr{F}_{3} & 1.9203+0.8564 \mathrm{i} & \mathscr{G}_{3} & -0.1165+0.0591 \mathrm{i} \\ \mathscr{F}_{4} & -0.7997+1.6044 \mathrm{i} & \mathscr{G}_{4} & -0.0454-0.0032 \mathrm{i}\end{array}$

TABLE 1. The values of the coefficients in (27).

Solving for $p_{3}$ and $p_{5}$ and substituting into (21) we obtain a condition for periodicity of the form

$$
2(\mathscr{B} \mathscr{D}-\mathscr{A} \mathscr{E}) \mathscr{J}=(\mathfrak{F} \mathscr{B}-\mathscr{C} \mathscr{E}) \mathscr{K}+(\mathscr{C} \mathscr{D}-\mathfrak{F} \mathscr{A}) \mathscr{L}
$$

where the quantities $\mathscr{A}-\mathfrak{F}, \mathscr{J}-\mathscr{L}$ are given by

$$
\begin{aligned}
& \mathscr{A}=G_{0}\left(9 \beta^{2}+\alpha^{2}\right)^{1 / 2}+9 \beta^{2}+\alpha^{2}, \quad \mathscr{B}=\frac{1}{2} G_{2}\left(25 \beta^{2}+\alpha^{2}\right)^{1 / 2}+\frac{5}{2} \beta H_{2}, \\
& \mathscr{C}=\frac{1}{2} \beta H_{2}-\frac{1}{2} \beta H_{4}-\frac{1}{2} G_{2}\left(\beta^{2}+\alpha^{2}\right)^{1 / 2}-\frac{1}{2} G_{4}\left(\beta^{2}+\alpha^{2}\right)^{1 / 2}, \\
& \mathscr{D}=\frac{1}{2} G_{2}\left(9 \beta^{2}+\alpha^{2}\right)^{1 / 2}-\frac{3}{2} \beta H_{2}, \quad \mathscr{E}=G_{0}\left(25 \beta^{2}+\alpha^{2}\right)^{1 / 2}+25 \beta^{2}+\alpha^{2}, \\
& \mathscr{J}=\frac{1}{2} \beta H_{4}-\frac{1}{2} G_{4}\left(\beta^{2}+\alpha^{2}\right)^{1 / 2}, \\
& \mathscr{J}=-\beta^{2}-\alpha^{2}-G_{0}\left(\beta^{2}+\alpha^{2}\right)^{1 / 2}-\frac{1}{2} \beta H_{2}-\frac{1}{2} G_{2}\left(\beta^{2}+\alpha^{2}\right)^{1 / 2}, \\
& \mathscr{K}=3 \beta H_{2}+3 \beta H_{4}+G_{4}\left(9 \beta^{2}+\alpha^{2}\right)^{1 / 2}, \quad \mathscr{L}=5 \beta H_{4}+G_{4}\left(25 \beta^{2}+\alpha^{2}\right)^{1 / 2} .
\end{aligned}
$$

In the following sections our choice of skin-friction profile $\lambda_{M}((17)$ or (20)) will allow us to write the coefficients $G_{0}, G_{2}, G_{4}, H_{2}, H_{4}$ in terms of the effective pressure amplitude $B$. Thus (26) can then be viewed as an amplitude equation to determine the evolution of $B(x)$.

\section{Determination of the amplitude equation}

We now wish to use our model profile to write the coefficients $G_{0}, G_{2}, G_{4}, H_{2}, H_{4}$ in terms of $B$ and rewrite the condition (26) for a periodic solution as an amplitude equation determining $B$ as a function of spanwise wavenumber $\beta$ and streamwise location $x$. In the model profile (17) for the wide-VWI we will take $x_{0}^{1 / 2}=\hat{\lambda}$, as in the numerical computations of $\S 2$, so that the factor $\hat{\lambda} / x_{0}^{1 / 2}$ is equal to unity. First we consider the functions $\mathscr{F}$ and $\mathscr{G}$. These quantities are expanded in Taylor series in $s$ about the neutral location $s=s_{N}$ say, where $s_{N}=\Omega / \alpha_{N}^{2 / 3}$, in view of the fact that the starting skin friction has been normalized to unity. It will prove necessary to retain terms up to quartic order so that the functions are represented in the form

$$
\mathscr{F}=\sum_{n=0}^{4} \mathscr{F}_{n}\left(s-s_{N}\right)^{n}, \quad \mathscr{G}=\sum_{n=0}^{4} \mathscr{G}_{n}\left(s-s_{N}\right)^{n} .
$$

The coefficients in the power series can be expressed in terms of Airy functions and their derivatives, but ultimately need to be evaluated numerically. The appropriate values are given in table 1 . Next, the quantity $s$ is written in terms of wavenumber $\alpha$ 
and the specific model profile $\lambda_{M}$. For convenience we adopt the notation

$$
\alpha=\alpha_{N} \hat{\alpha}, \quad \hat{x}=\frac{x-x_{0}}{x_{0}},
$$

so that at the start of the interaction $\hat{\alpha}=1$ and $\hat{x}=0$. In view of the fact that in the numerical simulations a singularity was often encountered relatively close to the start, we will assume $\hat{x}$ and $1-\hat{\alpha}$ to remain small throughout. It is convenient to define a new small parameter $\hat{e}$ such that

$$
\hat{\alpha}^{2 / 3}=1-\hat{e} .
$$

The quantity $s$ is then expanded in terms of $\hat{e}, \hat{x}$ and $B$ and terms up to quartic order in these small quantities are retained. Once $s$ has been expanded, $\mathscr{F}$ and $\mathscr{G}$ can also be expanded using (27), once again keeping terms up to quartic order. Since $G$ and $H$ are related to $\mathscr{G}$ and $\mathscr{F}$ via (22), it is then a straightforward matter to determine the coefficients $G_{0}, G_{2}, G_{4}, H_{2}, H_{4}$ defined in $\S 5$ in terms of $\beta, \alpha_{N}, \hat{x}, \hat{e}$ and $B . \dagger$

It is now possible to formulate the amplitude equation. Substitution of the expressions for $G_{0}, G_{2}, G_{4}, H_{2}, H_{4}$ into the condition (26) with retention of all terms up to quartic order yields an equation of the form

$$
\theta_{1} B^{4}+\left(\theta_{2} \hat{e}^{2}+\theta_{3} \hat{e} \hat{x}+\theta_{4} \hat{x}^{2}\right) B^{2}+\left(\theta_{5} \hat{e}^{4}+\theta_{6} \hat{e}^{3} \hat{x}+\theta_{7} \hat{e}^{2} \hat{x}^{2}+\theta_{8} \hat{e} \hat{x}^{3}+\theta_{9} \hat{x}^{4}\right)=0
$$

where the complex-valued coefficients $\theta_{1}-\theta_{9}$ are known functions of $\beta$. For a real solution for $B$ to exist therefore, the following two equations must be satisfied simultaneously:

$$
\begin{aligned}
f_{1}(B, \hat{e}, \hat{x}) \equiv & \theta_{1 r} B^{4}+\left(\theta_{2 r} \hat{e}^{2}+\theta_{3 r} \hat{e} \hat{x}+\theta_{4 r} \hat{x}^{2}\right) B^{2} \\
& +\left(\theta_{5 r} \hat{e}^{4}+\theta_{6 r} \hat{e}^{3} \hat{x}+\theta_{7 r} \hat{e}^{2} \hat{x}^{2}+\theta_{8 r} \hat{e} \hat{x}^{3}+\theta_{9 r} \hat{x}^{4}\right)=0, \\
f_{2}(B, \hat{e}, \hat{x}) \equiv & \theta_{1 i} B^{4}+\left(\theta_{2 i} \hat{e}^{2}+\theta_{3 i} \hat{e} \hat{x}+\theta_{4 i} \hat{x}^{2}\right) B^{2} \\
& +\left(\theta_{5 i} \hat{e}^{4}+\theta_{6 i} \hat{e}^{3} \hat{x}+\theta_{7 i} \hat{e}^{2} \hat{x}^{2}+\theta_{8 i} \hat{e} \hat{x}^{3}+\theta_{9 i} \hat{x}^{4}\right)=0,
\end{aligned}
$$

where the subscripts $r$ and $i$ denote the real and imaginary parts of the coefficients $\theta_{n},(n=1, \ldots, 9)$. For a given $\hat{x}$, these equations can be solved for the pressure amplitude $B$ and $\hat{e}$, the perturbation of the wavenumber away from its neutral value. Since (28) is a quadratic equation for $B^{2}$ we can now see why it was necessary to retain quartic terms in order to include the effects of nonlinearity.

\subsection{Solution for the wide-VWI using the appropriate model skin-friction profile}

Although in principle it is of course possible to solve the quadratic equations for $B^{2}$ exactly in an analytic form, the complicated nature of the coefficients $\theta_{n}$ renders this uninstructive. Instead, both numerical and graphical approaches to the solution prove informative, particularly since we are interested in why the solution ceases to exist beyond a critical $\hat{x}$-location. First we present in figure 5 results obtained by solving $f_{1}=f_{2}=0$ numerically. We show the results for $B(\hat{x})$ for $\beta=2$, the case where a singularity develops in the full computations. It is observed that the curve calculated is in excellent agreement with that obtained numerically in $\S 4$. This

$\dagger$ The expressions are of considerable length and are given for the model profile of $\S 4.1$ in full in Appendix B, which is available from the authors or the JFM Editorial Office. The expressions are of similar form and length for the model profile of $\S 4.2$. Although the process of deriving these coefficients is straightforward, it would not really have been feasible without the aid of an algebraic manipulation program (Mathematica in this case). 


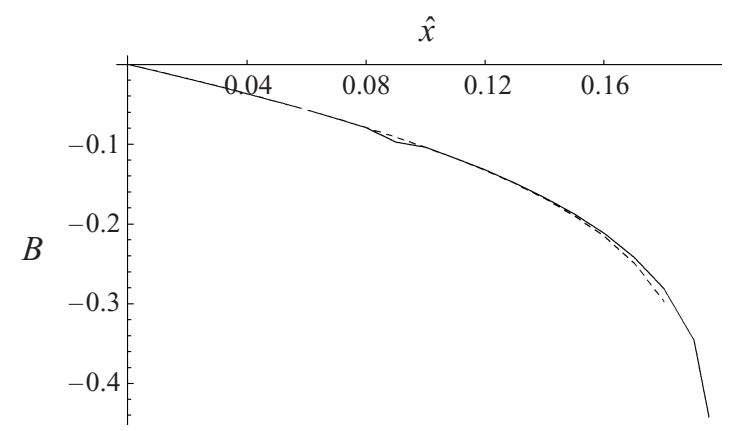

FIGURE 5. Comparison between the perturbation method (solid line) and the numerical solution of the pressure equation (dashed line) for the wide vortex of $\S 2$ with $\beta=2$.

indicates that our perturbation approach is valid even close to the $x$-location where the solution disappears, although the critical $\hat{x}$-value predicted by the perturbation approach is slightly greater than that obtained in $\S 4$. To understand the reason for the disappearance of the solution we consider the curves defined by $f_{1}$ and $f_{2}$ for fixed $\hat{x}$ and $\beta$ in $B, \hat{e}$ space. In figure $6(a)$ we plot the curves for the case $\beta=1, \hat{x}=0$. Since $B=\hat{e}=0$ at the location of wave input we expect the curves to intersect at the origin. It may be observed that the curves also intersect elsewhere but these other intersection points are not relevant to the physical problem. We now increase the value of $\hat{x}$ and follow the intersection point as it moves away from the origin. The sequence of figure $6(b-f)$ shows that there remains a solution up to and beyond values of $\hat{x}$ for which our perturbation approach is valid. This is consistent with the numerical results of $\S 2$ in which it was found that the pressure amplitude remained finite as $x$ was increased for a spanwise wavenumber $\beta$ equal to 1 . Figure $6(g-l)$ shows the corresponding situation for $\beta=2$. In this case it can be seen that the curves just touch when $\hat{x} \approx 0.2$ and then move apart as $\hat{x}$ is increased further. This is the point at which our solution disappears. This location can be determined more accurately in view of the fact that the Jacobian

$$
J=\frac{\partial f_{1}}{\partial B} \frac{\partial f_{2}}{\partial \hat{e}}-\frac{\partial f_{1}}{\partial \hat{e}} \frac{\partial f_{2}}{\partial B}
$$

vanishes at such a point. If for $\beta=2$ we solve the three equations $J=0, f_{1}=0, f_{2}=0$ simultaneously we obtain the following solution:

$$
\hat{x} \approx 0.1951, \quad B \approx-0.4612, \quad \hat{e} \approx 0.0778 .
$$

We see that the magnitude of $\hat{e}$ is substantially less than that of $B$ and $\hat{x}$ but nevertheless $B^{4}$ still remains relatively small, implying that a truncation at quartic order is still a reasonable approximation when the solution disappears. The breakdown location and the value of $\hat{e}$ predicted here are very close to those found in the full numerical simulation and are also in good agreement with the numerical calculations of the pressure equation carried out in $\S 4$ for this particular model profile.

\subsection{Solution for the wide-VWI under strong adverse pressure gradient using the model profile}

Figures 7 and 8 show the corresponding calculations for this slightly simplified interaction. First we observe from figure 7 that the computed solutions for $B(\hat{x})$ compare well with those obtained by numerically solving the pressure equation in $\S 4$. As in $\S 6.1$, the critical $\hat{x}$-value is slightly greater using the perturbation approach. 

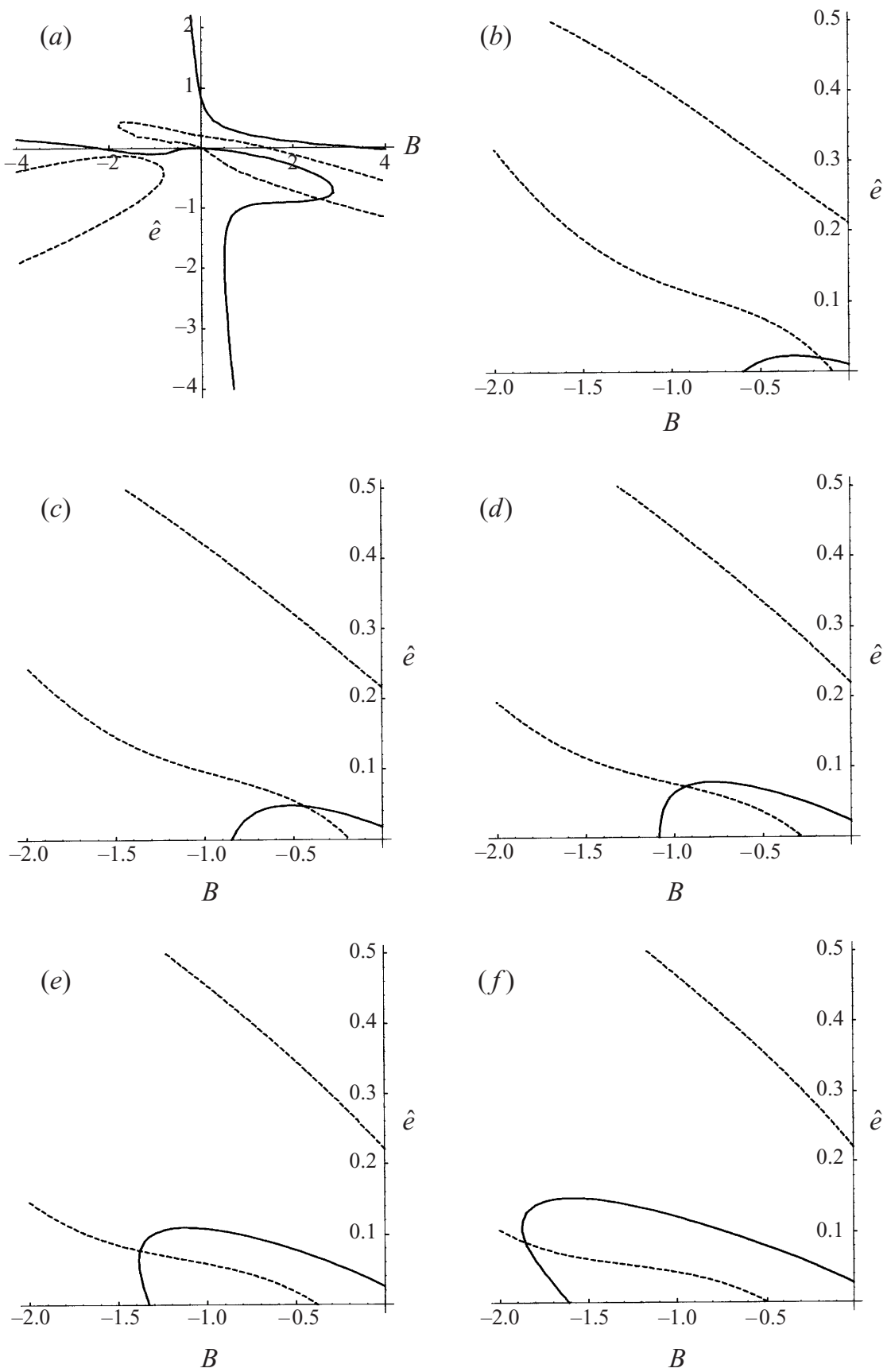

FIGURE $6(a-f)$. For caption see facing page.

Figure 8 shows the $f_{1}$ and $f_{2}$ curves in $B, \hat{e}$ space. As before the solution starts from the origin when $\hat{x}=0$ and moves into the second quadrant as $\hat{x}$ increases. The case $\beta=1$ is presented here: similar results were found for all other values of $\beta$ we tried. In all cases there exists a critical value of $\hat{x}$ beyond which there is no real solution for $B$ and $\hat{e}$. The critical solutions for various $\beta$ are given in table 2 . It could be argued 

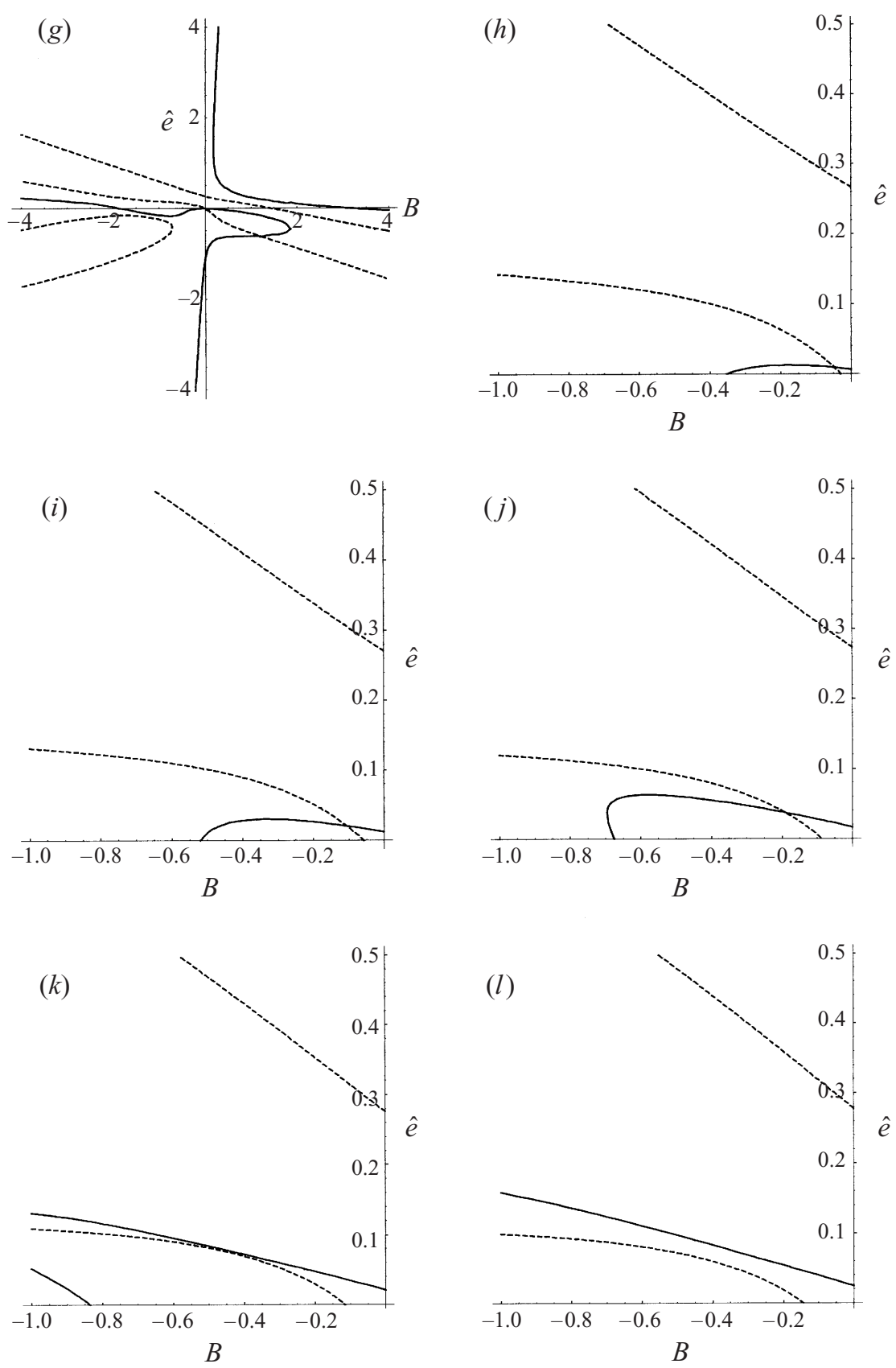

FIgURE 6. Perturbation results for the wide vortex of $\S 2$ with model skin friction. The curves $f_{1}(B, \hat{e})$ (solid lines) and $f_{2}(B, \hat{e})$ (dashed lines) are shown in the $(B, \hat{e})$-plane for fixed $\hat{x} .(a-f)$ Spanwise wavenumber $\beta=1$ and the following values of $\hat{x}:(a) \hat{x}=0,(b) 0.1,(c) 0.2,(d) 0.3,(e) 0.4,(f) 0.5$. $(g-l) \beta=2$ and the following values of $\hat{x}:(g) 0,(h) 0.05,(i) 0.1,(j) 0.15,(k) 0.2,(l) 0.25$.

that for $\beta=1$, the value of $B$ is sufficiently large that our perturbation approach is not valid: nevertheless there is good agreement once again with the full numerical simulations described in $\S 3$ and the computations of the pressure equation presented in $\S 4$. 


\begin{tabular}{lcccc}
\hline$\beta$ & $\hat{x}$ & $B$ & $\hat{e}$ \\
1 & 0.1479 & -0.5481 & 0.0600 \\
1.5 & 0.1453 & -0.3246 & 0.0625 \\
2 & 0.1444 & -0.3110 & 0.0629
\end{tabular}

TABLE 2. The critical solutions for various $\beta$.

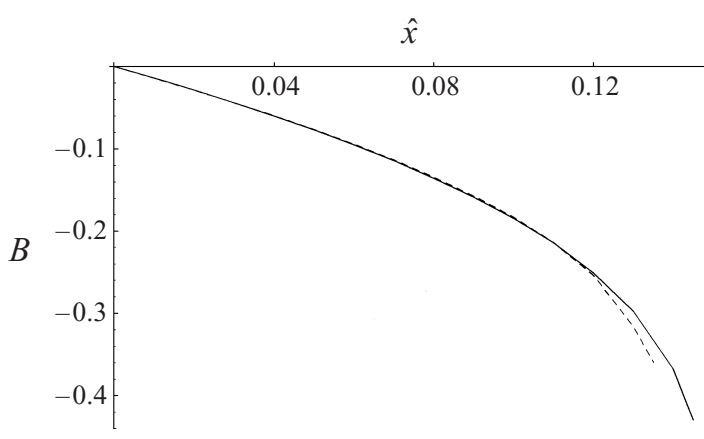

FiguRE 7. Comparison between the perturbation method (solid line) and the numerical solution of the pressure equation (dashed line) for the VWI subject to a strong adverse pressure gradient $(\S 3)$ with $\beta=1$.

\section{Further applications of the perturbation approach}

\subsection{Alternative pressure-displacement laws}

Although we have concentrated in this paper on flows governed by the incompressible TS pressure-displacement law (8), we have also carried out an investigation of the effects on our results of using other $p-A$ laws. For example, for the case of the stability of a wall jet the VWI equations remain unchanged except that (8) is replaced by the much simpler form

$$
p=\alpha^{2} A .
$$

See for example Smith (1988). The pressure equation (6) can then be transformed into the equation

$$
r^{\prime \prime}+\left(\frac{1}{2}\left(\frac{\lambda^{\prime}}{\lambda} \mathscr{F}(s)\right)^{\prime}-\frac{1}{4}\left(\frac{\lambda^{\prime}}{\lambda} \mathscr{F}(s)\right)^{2}-\left(\alpha^{2}+\alpha^{-1 / 3} \lambda^{5 / 3} \mathscr{G}(s)\right)\right) r=0,
$$

where

$$
r(x, z)=\exp \left(-\frac{1}{2} \int_{0}^{z} \frac{\lambda^{\prime}}{\lambda} \mathscr{F}(s(x, z)) \mathrm{d} z\right) p(x, z),
$$

and ' denotes differentiation with respect to $z$. Either of the model profiles for $\lambda$ proposed in $\S 4$ can be substituted into (29) which as a result reduces to the Whittaker-Hill equation:

$$
r^{\prime \prime}+\left(t_{0}+2 t_{2} \cos 2 \beta z+2 t_{4} \cos 4 \beta z\right) r=0
$$

(Magnus \& Winkler 1966). As before, the constants $t_{0}-t_{4}$ can be written in terms of the perturbation quantities $B, \hat{e}$ and $\hat{x}$. We find that $t_{0} \sim O(1), t_{2} \sim O(\hat{x})$ and 

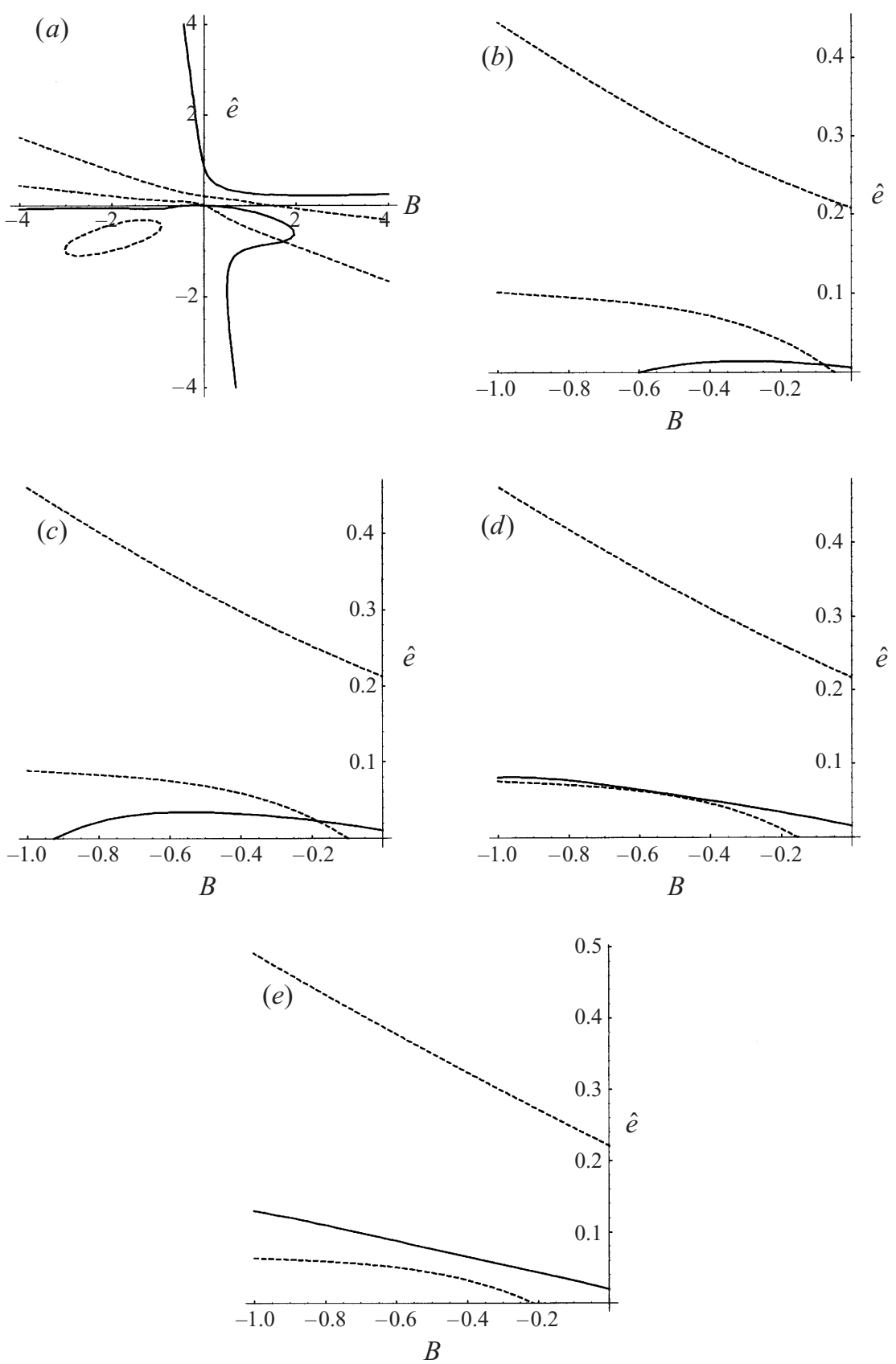

FIGURE 8. Perturbation results for adverse pressure gradient VWI with model skin friction. The curves $f_{1}(B, \hat{e})$ (solid lines) and $f_{2}(B, \hat{e})$ (dashed lines) are shown in the $(B, \hat{e})$-plane for fixed $\hat{x}$ and a spanwise wavenumber $\beta=1$. (a) $\hat{x}=0,(b) 0.05,(c) 0.1,(d) 0.15$, (e) 0.2 .

$t_{4} \sim O\left(t_{2}^{2}\right)$. The analogous expression to (26), ensuring the existence of a periodic solution to (30) (with leading-order term $\cos \beta z$ ), is

$$
t_{0}=\beta^{2}-t_{2}-\frac{t_{2}^{2}}{8 \beta^{2}}-\left(\frac{t_{2} t_{4}}{4 \beta^{2}}-\frac{t_{2}^{3}}{64 \beta^{4}}\right)-\left(\frac{t_{2}^{4}}{1536 \beta^{6}}-\frac{t_{4} t_{2}^{2}}{48 \beta^{4}}+\frac{t_{4}^{2}}{6 \beta^{2}}\right),
$$


where we have retained terms up to quartic order. As in $\S 6$, substitution of the appropriate expressions for $t_{0}, t_{2}, t_{4}$ leads to an amplitude equation of the form (28). This can be analysed in a similar way to that outlined in $\S 6$, and similar results are obtained to the previous cases considered. Once again we find a critical value of $\hat{x}$ beyond which no solution for $B$ is possible.

The main reason for mentioning these results here is that they indicate that the precise form of the $p-A$ law is not a crucial factor in the formation of the finitedistance singularity. On the basis of the studies we have carried out we would expect the same type of behaviour to occur in the wide-VWI equations subject to any neutralwave-supporting $p-A$ law (although possibly only for a limited range of spanwise wavenumbers).

\subsection{Marginally-separating flows}

WBS also considered the effects of the wide-VWI on a marginally-separating flow. They show that equation (14) for the skin friction is modified to

$$
\lambda \frac{\partial \lambda}{\partial \hat{x}}=\frac{1}{2}(\hat{x}-1)+\frac{\partial w_{s}}{\partial z},
$$

with all other equations remaining unchanged. The appropriate starting condition is

$$
\lambda=\lambda_{I} \quad \text { at } \hat{x}=0
$$

where $\lambda_{I}$ is a constant which describes the variation of some parameter (e.g. angle of attack of an airfoil) controlling separation. Numerical solutions of the governing equations indicate that for $\lambda_{I} \lesssim 2.5$, the interaction terminates in a finite-distance blow-up of the type described in $\S 3$. A perturbation analysis of the type described in $\S \S 5$ and 6, using a suitable model skin-friction profile, yields similar results to those mentioned earlier, and the critical value of $\hat{x}$ can be predicted quite accurately using this approach. For $\lambda_{I}>2.5$, the numerical solutions suggest that the interaction persists indefinitely downstream on the lengthscale considered here. The corresponding perturbation approach fails to find a critical $\hat{x}$-location as expected in this case (cf. $\S 6.1, \beta=1$ ).

\section{Conclusions}

The main conclusions of our study are as follows.

Numerical solutions of the wide-VWI equations ( $(2)$ indicate that a two-dimensional Blasius boundary layer can be rendered three-dimensional by the introduction of a three-dimensional TS wave close to the appropriate neutral position. For a spanwise wavenumber $\beta=1$, the mean flow becomes strongly three-dimensional while the wave amplitude oscillates. For $\beta=2$ the behaviour is much more dramatic, with a singularity developing in the wave pressure amplitude within a short distance from the position of wave input. The singularity is similar to that observed in the computations of WBS for a VWI close to separation. The rapid formation of a singularity is not inconsistent with transition experiments involving three-dimensional waves: for example, Kovasznay et al. (1962) find that the evolution from ribbon excitation to transition occupies only ten wavelengths in their experiment.

By using a simplified expression for the streamwise mean flow skin friction, involving only one parameter $B$, it is possible to make use of the proximity of the singularity location to that of wave input and to formulate an algebraic equation to be satisfied by $B$ and the correction to the neutral wavenumber $\hat{e}$. By solving this equation the 
singularity location can be found accurately and may be interpreted geometrically in terms of the intersection of curves in the $(B, \hat{e})$-plane. The fact that the singularity can be modelled in this way illustrates that it is mathematical rather than an artifact of a numerical scheme. These results also indicate that the region of parameter space over which the pressure equation (6) admits periodic solutions is quite restricted.

Given that the critical $x$-location, $x_{c}$ say, is achieved when the curves $f_{1}$ and $f_{2}$ just touch, the behaviour of $B$ as this point is approached must be of the form

$$
B \sim B_{0}+\left(x_{c}-x\right)^{1 / 2} B_{1}
$$

as $x \rightarrow x_{c}-$, in view of the quadratic nature of equation (28). Making use of the relationship between $B$ and the pressure amplitude $|q|$ noted in $\S 4$, we obtain the predictions

$$
|q| \propto\left(x_{c}-x\right)^{-1 / 12} \text { as } x \rightarrow x_{c}-,
$$

for the wide vortex of $\S 2$, and

$$
|q| \propto\left(x_{c}-x\right)^{-1 / 4} \quad \text { as } x \rightarrow x_{c}-,
$$

for the adverse pressure gradient VWI of $\S 3$. The latter of these asymptotic forms is in agreement with the singularity structure proposed in Walton et al. (1994), while the former, rather weaker, singularity is in line with the numerical results of $\S 2$ for the $\beta=2$ case. The weakness of this singularity goes some way to explaining the suddenness of the disappearance of the solution for $|q|$ noted in $\S 2$. The blow-up of the pressure amplitude implies a spanwise slip velocity proportional to $\left(x_{c}-x\right)^{-1 / 6}$ and suggests that the wide-VWI acquires a two-tiered terminal structure. In the lower of the layers the solution is of similarity form with

$$
\begin{gathered}
u=\left(x_{c}-x\right)^{1 / 3} \lambda_{0}(z) \eta+\left(x_{c}-x\right)^{5 / 6} u_{1}(\eta, z)+\ldots, \\
v=\left(x_{c}-x\right)^{1 / 6} v_{1}(\eta, z)+\ldots, \\
w=\left(x_{c}-x\right)^{-1 / 6} w_{1}(\eta, z)+\ldots, \\
\eta=y /\left(x_{c}-x\right)^{1 / 3},
\end{gathered}
$$

as $x \rightarrow x_{c}-$. Here the shear function $\lambda_{0}(z)$ is determined by the solution in the upper layer to be discussed presently. By substituting these expansions into the wide-VWI equations (1), (2), we obtain the following leading-order balances:

$$
\begin{gathered}
\frac{1}{3} \eta \frac{\partial u_{1}}{\partial \eta}-\frac{5}{6} u_{1}+\frac{\partial v_{1}}{\partial \eta}+\frac{\partial w_{1}}{\partial z}=0, \\
\lambda_{0}(z)\left(\frac{1}{3} \eta^{2} \frac{\partial u_{1}}{\partial \eta}-\frac{5}{6} \eta u_{1}+v_{1}\right)+\lambda_{0}^{\prime}(z) \eta w_{1}=\frac{\partial^{2} u_{1}}{\partial \eta^{2}}, \\
\lambda_{0}(z)\left(\frac{1}{3} \eta^{2} \frac{\partial w_{1}}{\partial \eta}+\frac{1}{6} \eta w_{1}\right)=\frac{\partial^{2} w_{1}}{\partial \eta^{2}} .
\end{gathered}
$$

The equations are subject to matching conditions with the upper layer as $\eta \rightarrow \infty$, and the spanwise slip condition on $\eta=0$ arising from the TS wave forcing. Suitable solutions exist: for example, the solution for $w_{1}$ can be written in terms of the 


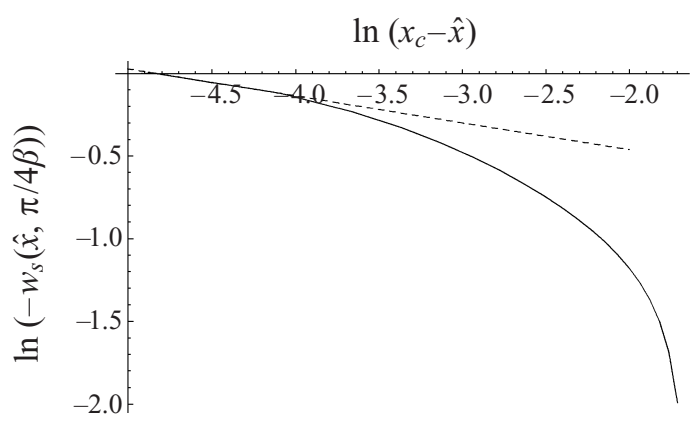

FIGURE 9. A comparison of perturbation theory and full numerical solution for the wide VWI of $\S 2$ with $\beta=2$. Vertical axis: $\ln \left(-w_{s}(\hat{x}, \pi / 4 \beta)\right)$; horizontal axis: $\ln \left(x_{c}-\hat{x}\right)$ with $x_{c}=0.189$. The solid curve represents results from a full numerical simulation, while the dashed line has the theoretically-predicted gradient of $-\frac{1}{6}$.

Whittaker function $\mathscr{W}$ as

$$
w_{1}(\eta, z) \propto \frac{1}{\eta} \mathrm{e}^{\lambda_{0}(z) \eta^{3} / 18} \mathscr{W}_{1 / 6,1 / 6}\left(\lambda_{0}(z) \eta^{3} / 9\right) .
$$

The decay of $w_{1}$ is proportional to $\eta^{-1 / 2}$ as $\eta \rightarrow \infty$, and this leads to an $O(1)$ spanwise contribution in the upper layer where $y$ is of $O(1)$. The full velocity expansions in this region are

$$
\begin{aligned}
& u=\hat{U}_{0}(y, z)+\left(x_{c}-x\right) \hat{U}_{1}(y, z)+\ldots, \\
& v=\hat{V}_{0}(y, z)+\ldots, w=\hat{W}_{0}(y, z)+\ldots .
\end{aligned}
$$

Here the leading-order streamwise profile $\hat{U}_{0}(y, z)$ is unknown and determined in practice by the flow development upstream of the singularity. We require

$$
\hat{U}_{0} \rightarrow 1 \text { as } y \rightarrow \infty, \quad \hat{U}_{0} \sim \lambda_{0}(z) y \text { as } y \rightarrow 0,
$$

to satisfy the outer boundary condition (4) and to match with the inner solution described above. More evidence supporting this singularity structure is presented in figure 9 where we compare log-log plots of the spanwise slip velocity from theory and computation. In contrast to previous proposed singularity structures for the VWI equations (e.g. Smith \& Walton 1989; Walton \& Smith 1992), we have demonstrated that a singularity does indeed occur in the governing equations when they are solved numerically. In addition our approach enables us to obtain a prediction for $x_{c}(\beta)$ without the need for a full numerical simulation for each value of $\beta$.

Our investigations of other mean flows and different pressure-displacement laws suggest that the formation of a singularity in these equations should be considered the norm rather than the exception. Although the modelling of the development of the flow beyond the singularity position is beyond the scope of the present work we can see that if the wave amplitude continues to rise, the disturbance will eventually become comparable with the induced vortex shear within the lower deck. Before this stage is reached however, the mean flow lengthscale has contracted sufficiently that it has become comparable with the TS wavelength. Explicit $x$-dependence is then incorporated into the pressure equation and the resulting set of governing equations can no longer simply be marched forward due to the enhanced ellipticity now present in the system. Further work is required to elucidate the fine details of this new structure. 


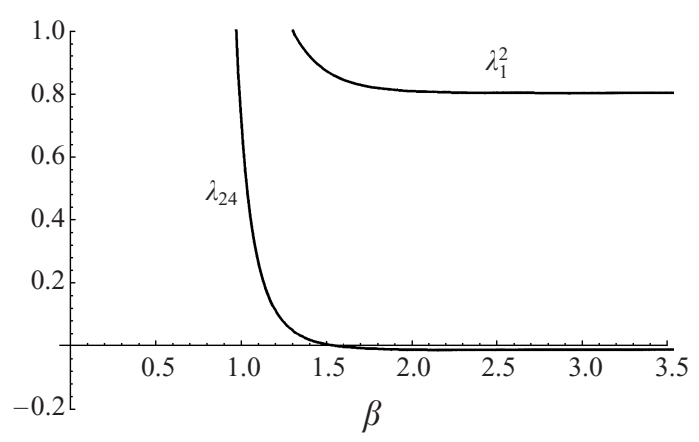

FIGURE 10. A comparison of the skin-friction coefficients $\lambda_{1}^{2}, \lambda_{24}$ plotted versus spanwise wavenumber $\beta$.

\section{Appendix. Concerning the mathematical validity of the approximation of the skin friction by a model profile}

Although the model profiles (17), (20) neglect the existence of higher harmonics $(\propto \cos 4 \beta z)$ that strictly speaking are present in the full numerical calculations, there is a critical value of the spanwise wavenumber close to which (17), (20) constitute rational approximations to the full nonlinear problem to the order considered. We illustrate this here for the adverse pressure gradient VWI of $\S 3$, with the argument for the wide-VWI following similar lines.

Reconsidering the initial development of the VWI, we see from equations (5), (6) and (19) that the skin friction develops according to

$$
\begin{aligned}
\lambda^{2}= & 1-(x+1)+(x+1) \lambda_{1} \cos 2 \beta z \\
& +(x+1)^{2}\left(\lambda_{20}+\lambda_{22} \cos 2 \beta z+\lambda_{24} \cos 4 \beta z\right)+O(x+1)^{3},
\end{aligned}
$$

where $x=-1$ is the position of wave input. The coefficients of relevance to the present argument are $\lambda_{1}$ and $\lambda_{24}$, and they can be written in the form

$$
\begin{gathered}
\lambda_{1}=-\frac{4 \beta^{2}}{\alpha_{0}^{2}}\left|Q_{0}\right|^{2}\left(\beta^{2}-\alpha_{0}^{2}\right), \\
\lambda_{24}=-\frac{8 \beta^{4}}{\alpha_{0}^{4}}\left|Q_{0}\right|^{4}\left(\beta^{2}-\alpha_{0}^{2}\right)\left(\beta^{2}-\alpha_{0}^{2}-\frac{\left(3 \beta^{2}-\alpha_{0}^{2}\right)\left(q_{1} \beta^{2}+q_{2} \alpha_{0}^{2}\right)}{9 \beta^{2}+\alpha_{0}^{2}-\left(\beta^{2}+\alpha_{0}^{2}\right)^{1 / 2}\left(9 \beta^{2}+\alpha_{0}^{2}\right)^{1 / 2}}\right) .
\end{gathered}
$$

Here, the quantity $Q_{0}$ is a complex-valued amplitude function that can be determined as a function of $\beta$ (see Walton et al. 1994), $\alpha_{0}$ is the corresponding neutral wavenumber, and the quantities $q_{1}, q_{2}$ are real constants with

$$
q_{1} \simeq 1.923, \quad q_{2} \simeq 1.169 .
$$

Our theory assumes that terms proportional to $\cos 4 \beta z$, occurring for example in (23), (24) are generated purely from the interaction of $\cos 2 \beta z$ terms in the model expression (20) for the skin friction. This amounts to the assumption that

$$
\lambda_{24} \ll O\left(\lambda_{1}^{2}\right) \text {. }
$$

Figure 10 shows the coefficients $\lambda_{1}^{2}, \lambda_{24}$ plotted versus spanwise wavenumber $\beta$. Although this inequality cannot be satisfied for all $\beta$, there exists a critical value, $\beta_{c}$ say, at which $\lambda_{24}\left(\beta_{c}\right)=0$. Provided $\beta$ is close to $\beta_{c}$, the inequality is satisfied and the model profile then provides the appropriate asymptotic approximation to the full 
problem. For the adverse pressure gradient VWI, figure 10 shows that $\beta_{c} \simeq 1.5$ with this value being close to that at which the numerical solutions and the perturbation approach agree best according to our results.

\section{REFERENCES}

Ainara, Y., Tomita, Y. \& ITo, A. 1984 Generation, development and distortion of longitudinal vortices in boundary layers along concave and flat plates. In Laminar-Turbulent Transition (ed. V. V. Kozlov), pp. 447-454. Springer.

Benney, D. J. 1984 The evolution of disturbances in shear flows at high Reynolds numbers. Stud. Appl. Maths 70, 1-19.

BENNEY, D. J. \& CHOw, K. 1985 An alternative approach to nonlinear instabilities in hydrodynamics. Stud. Appl. Maths 73, 261-267.

BlaCKabY, N. D. 1994 Tollmien-Schlichting/vortex interactions in compressible boundary layer flows. IMA J. Appl. Maths 53, 191-214.

Brown, S. N. \& Smith, F. T. 1996 On vortex/wave interactions. Part 1. Non-symmetrical input and cross-flow in boundary layers. J. Fluid Mech. 307, 101-133.

Cowley, S. J. \& Wu, X. 1994 Asymptotic approaches to transition modelling. In Progress in Transition Modelling. AGARD Rep. 793, 3: pp. 1-38.

Davis, D. A. R. \& Smith, F. T. 1994 Influence of cross-flow on nonlinear Tollmien-Schlichting/ vortex interaction. Proc. R. Soc. Lond. A 446, 319-340.

Goldstein, M. E. \& Wundrow, D. W. 1995 Interaction of oblique instability waves with weak streamwise vortices. J. Fluid Mech. 284, 377-407.

GoldsteIn, S. 1948 On laminar boundary-layer flow near a point of separation. Q. J. Mech. Appl. Maths 1, 43-69.

Hall, P. \& Smith, F. T. 1988 The nonlinear interaction of Tollmien-Schlichting waves and TaylorGörtler vortices in curved channel flows. Proc. R. Soc. Lond. A 417, 255-282.

Hall, P. \& SMith, F. T. 1989 Nonlinear Tollmien-Schlichting/vortex interaction in boundary layers. Eur. J. Mech. B 8, 179-205.

Hall, P. \& Smith, F. T. 1991 On strongly nonlinear vortex/wave interactions in boundary-layer transition. J. Fluid Mech. 227, 641-666.

Healey, J. J. 1995 On the neutral curve of the flat plate boundary layer: comparison between experiment, Orr-Sommerfeld theory and asymptotic theory. J. Fluid Mech. 288, 59-73.

Herbert, T. 1997 Parabolized stability equations. Ann. Rev. Fluid Mech. 29, 245-283.

Klebanoff, P. S., Tidstrom, K. D. \& Sargent, L. M. 1962 The three-dimensional nature of boundary-layer instability. J. Fluid Mech. 12, 1-34.

KLEISER, L. \& ZANG, T. A. 1991 Numerical simulation of transition in wall-bounded shear flows. Ann. Rev. Fluid Mech. 23, 495-537.

Kovasznay, L. S., Komoda, H. \& Vasudeva, B. R. 1962 In Proc. 1962 Heat Transfer and Fluid Mechanics Institute, pp. 1-26. Stanford University Press.

Magnus, W. \& Winkler, S. 1966 Hill's Equation. Interscience.

PATEL, R. A. 1997 Theory and computation of nonlinear vortex-wave interactions in internal and external flows. PhD thesis, University of London.

ReED, H. L. 1994 Direct numerical simulation of transition: the spatial approach. In Progress in Transition Modelling. AGARD Rep. 793, 6: pp. 1-46.

REYNOLDS, O. 1883 An experimental investigation of the circumstances which determine whether the motion of water will be direct or sinuous, and of the law of resistance in parallel channels. Phil. Trans. R. Soc. Lond. A 174, 935-982.

Sмith, F. T. 1979 Nonlinear stability of boundary layers for disturbances of various sizes. Proc. R. Soc. Lond. A 368, 573-589.

Smith, F. T. 1988 A reversed-flow singularity in interacting boundary layers. Proc. R. Soc. Lond. A 420, 21-52.

Smith, F. T. \& Blennerhassett, P. 1992 Nonlinear interaction of oblique three-dimensional Tollmien-Schlichting waves and longitudinal vortices, in channel flows and boundary layers. Proc. R. Soc. Lond. A 436, 585-602. 
Smith, F. T., Brown, S. N. \& Brown, P. G. 1993 Initiation of three-dimensional nonlinear transition paths from an inflectional profile. Eur. J. Mech. B 12, 447-473.

Smith, F. T. \& Walton, A. G. 1989 Nonlinear interaction of near-planar TS waves and longitudinal vortices in boundary-layer transition. Mathematika 36, 262-289.

Stewart, P. A. \& Smith, F. T. 1992 Three-dimensional nonlinear blow-up from a nearly planar initial disturbance, in boundary layer transition: theory and experimental comparisons. J. Fluid Mech. 244, 79-100.

Walton, A. G. 1996 Strongly nonlinear vortex-Tollmien-Schlichting-wave interactions in the developing flow through a circular pipe. J. Fluid Mech. 319, 77-107.

Walton, A. G., Bowles, R. I. \& SMith, F. T. 1994 Vortex-wave interaction in separating flows. Eur. J. Mech. B 13, 629-655 (referred to herein as WBS).

Walton, A. G. \& Smith, F. T. 1992 Properties of strongly nonlinear vortex/Tollmien-Schlichtingwave interactions. J. Fluid Mech. 244, 649-676.

Wray, A. \& Hussaini, M. Y. 1984 Numerical experiments in boundary-layer stability. Proc. R. Soc. Lond. A 392, 373-389.

Wu, X., LeE, S. S. \& Cowley, S. J. 1993 On the weakly nonlinear three-dimensional instability of shear layers to pairs of oblique waves: the Stokes layer as a paradigm. J. Fluid Mech. 253, $681-721$.

Wu, X., Stewart, P. A. \& Cowley, S. J. 1996 On the weakly nonlinear development of TollmienSchlichting wave-trains in boundary layers. J. Fluid Mech. 323, 133-171. 